\title{
EXTREMAL PROBLEMS OF DISTANCE GEOMETRY RELATED TO ENERGY INTEGRALS
}

\author{
BY \\ RALPH ALEXANDER AND KENNETH B. STOLARSKY(1)
}

\begin{abstract}
ABSTRAC.T. Let $K$ be a compact set, $M$ a prescribed family of (possibly signed) Borel measures of total mass one supported by $K$, and $f$ a continuous real-valued function on $K \times K$. We study the problem of determining for which $\mu$ $\epsilon \Pi$ (if any) the energy integral $I(K, \mu)=\int_{K} \int_{K} f(x, y) d \mu(x) d \mu(y)$ is maximal, and what this maximum is. The more symmetry $K$ has, the more we can say; our results are best when $K$ is a sphere. In particular, when $M$ is atomic we obtain good upper bounds for the sums of powers of all $\left(\begin{array}{l}n \\ 2\end{array}\right)$ distances determined by $n$ points on the surface of a sphere. We make use of results from Schoenberg's theory of metric embedding, and of techniques devised by Pólya and Szegö for the calculation of transfinite diameters.
\end{abstract}

1. Background and summary of results. In this paper we will investigate a number of extremal problems in distance geometry. Our work is in many ways analogous to the study of energy integrals in classical potential theory.

Let $K$ be a compact set in a Euclidean space and $\pi$ be a prescribed family of Borel measures (possibly signed) of total mass one supported by $K$. Suppose $f$ is a continuous real-valued function on $K \times K$. We consider the family of integrals having the form

$$
I(K, \mu)=\iint f(x, y) d \mu(x) d \mu(y), \quad \mu \in \mathbb{M} .
$$

A number of interesting questions naturally arise concerning $I(K)$, the supremum of the numbers $I(K, \mu)$ with $\mu$ in $\pi$ :

(i) What is the numerical value of $I(K)$ ?

(ii) Does there exist a $\mu_{0}$ in $\pi$ such that $I\left(K, \mu_{0}\right)=I(K)$ ?

(iii) If $\mu_{0}$ exists, is this measure unique?

(iv) Can an extremal measure $\mu_{0}$ be explicitly produced?

Received by the editors March 20, 1973 and, in revised form, April 20, 1973.

AMS (MOS) subject classifications (1970). Primary 52A25, 52A40.

Key words and phrases. Extremal problems of distance geometry, energy integrals, family of signed Borel measures, Euclidean $m$-sphere, great circle distance, ultraspherical harmonics, metric embedding, strictly metrically homogeneous, transfinite diameter, metric curvature.

(1) The research of this author was supported in part by the National Science Foundation, under contract no. GP-12363. 
Let us give an example. Suppose $K$ is a unit Euclidean $m$-sphere and $\pi$ is the collection of all atomic measures on $K$ consisting of $n$ atoms of weight $1 / n$. Let $f(x, y)=|x-y|$. It is easily seen that $n^{2} I(K)$ is the supremum of all sums $\Sigma_{i, j}\left|p_{i}-p_{j}\right|$, where $p_{1}, \cdots, p_{n}$ are variable points on the sphere. We remark that if $m>1$ and $n>m+2$, no methods exist for answering completely questions (i), (iii), and (iv). If in this example $f(x, y)$ is changed to be the great circle distance from $x$ to $y$, Kelly [17], Nielson [23] and Sperling [31] have shown that $I(K)$ $=\pi / 4$ ( $n$ even).

When (as above) $M$ consists of the positive measures containing $n$ atoms of weight $1 / n$, we call $\pi$ the $n$-discrete family.

G. Björck [2], by using the elegant methods of potential theory, has investigated the case where $K$ is a compact set in a Euclidean space, $\mathbb{M}$ consists of the positive Borel measures of mass one supported by $K$, and $f(x, y)=|x-y|^{\lambda}$ where $0<\lambda$. He showed that if $0<\lambda<2$, then $\mu_{0}$ exists and is unique. His paper also contains a nice discussion of the cases $\lambda=2$ and $\lambda>2$.

The classical paper of I. Schur [30] treats the case where $K$ is an interval, $f(x, y)=\log |x-y|$, and $\pi$ is the $n$-discrete family. It brilliantly answers all four of our questions.

L. Fejes Tóth [7] points out that if $K$ is a circle and if $f$ has strong convexity properties, one can employ a winding number argument to produce an extremal $n$-discrete measure $\mu_{0}$. Since we are very interested in Euclidean spheres, we wish to know as much as possible about the circle. However, even here, there are unanswered questions.

One must also mention the important work of Pólya and Szegö [24]. By expanding $|x-y|^{\lambda}$ in spherical harmonics they were able to obtain much information about the case where $K$ is a 2 -sphere. We find their technique extremely interesting and worthy of further investigation.

We now give a brief summary of our present work.

In $\$ 2$ we take a close look at Fejes Tóth's method for the circle and identify a rather general class of functions for which the method is effective. As a nontrivial example, we prove that any elementary symmetric function of the $\left(\begin{array}{l}n \\ 2\end{array}\right)$ distances determined by $n$ points on a circle is uniquely maximized when the points are the distinct vertices of a regular $n$-gon. Analogous results for non-Euclidean circles are indicated.

In $\$ 3$ we show that the results of I. J. Schoenberg ([25]-[28]) on metric embedding are powerful tools for investigating our problems. For example, if $K$ is a finite set in a Euclidean space, $f(x, y)=|x-y|^{\lambda}$ with $0<\lambda<2$, and $\pi$ consists of all signed Borel measures of mass one supported by $K$, we show that $\mu_{0}$ is unique and can be computed directly. In this case we show that $I(K)$ has a 
nice geometrical interpretation in terms of the radius of a certain sphere. This allows application of our results to certain problems which, by their statements, do not seem closely related to extremal problems.

For example, Grünbaum and Kelly [9] call a set of points $\left\{p_{1}, \cdots, p_{n}\right\}$ in $E^{m}$ strictly metrically bomogeneous if the set (counting multiplicities) of distances $\left\{\left|p_{i}-p_{1}\right|, \cdots,\left|p_{i}-p_{n}\right|\right\}$ is independent of $i$. (The vertex sets of regular polygons and Platonic solids give examples of such sets.) Their characterization of such planar sets shows that when $m=2$, the points $p_{1}, \cdots, p_{n}$ lie on a circle centered at their centroid. We generalize this to arbitrary $m$ by showing that the set must lie on a sphere centered at the centroid.

Also in $\S 3$, it is shown that if $K$ is a compact set in $E^{m}, f(x, y)=|x-y|^{\lambda}$ with $0<\lambda \leq 1$, and $\mathbb{N}$ is the family of signed Borel measures of total mass one supported by $K$, then $I(K) \leq c$. Diameter $K$ where $c$ depends only on $m$. The number $I(K)$ is interpreted in terms of the radius of a certain Hilbert sphere associated with a metric embedding.

In $\$ 4$ we assume that $K$ is a Euclidean sphere, $f$ satisfies a certain definiteness condition, and $\pi$ is the $n$-discrete family. We show that $c(1-1 / n) \leq I(K) \leq$ $c$ where the constant $c$ depends only on $f$. We also show that the great circle metric, as well as the Euclidean metric, satisfies the definiteness condition. This generalizes the previously cited work of Nielson and Sperling.

In $\$ 5$ we expand upon the method of Pólya and Szegö in the situation where $K$ is a Euclidean $m$-sphere, $f(x, y)=|x-y|^{\lambda}$ with $0<\lambda \leq 2$, and $\pi$ is the $\dot{n}$ discrete family. We show for $m \geq 2$ that $n^{2} I(K) \leq c(m, \lambda)_{n}^{2}-b(m, \lambda)_{n}-\gamma(m, \lambda)$. Here $c(m, \lambda)$ is the "constant of uniform distribution," $b(m, \lambda)>0$, and $\gamma(m, \lambda)=$ $(2 \lambda+1) / m$. The previously cited work of Björck shows that $I(K) \leq c(m, \lambda)$. To obtain our inequality, the method of Pólya and Szegö is extended to ultraspherical harmonics, and further refined. For $\lambda=1$ very good estimates of $I(K)$ are available ([1], [33]); for $0<\lambda \leq 1$ see also [32].

In $\$ 6$ various constants (which do not seem to appear in the literature) related to transfinite diameter and curvature are computed. Hille [12] shows that the transfinite diameter of the Hilbert sphere is $\sqrt{2}$; we show by explicit formulae that the transfinite diameter of the $m$-sphere is $\sqrt{2}+O(1 / m)$. Also, a new metric curvature is introduced. We remark that Hille's recent book [13] contains a wealth of material on transfinite diameters.

Throughout the paper we often use notations of convenience, rather than $l, K$, and $\pi$. For example, in $\$ 4$ the expression $n^{2} I(K)$ becomes $S(f, n)$.

2. Extensions of Fejes Tóth's work on the circle. In this section we extend Fejes Tóth's results for the unit circle to a more general class of functions. In order to reserve the letters $i, j, k$ for integers we write $\exp (\theta)$ instead of 
$\exp (i \theta)$. To obtain an appropriate level of generality, we shall study functions defined on angle tuples $\left(\theta_{0}, \theta_{1}, \ldots, \theta_{r}\right)$ rather than on point tuples $\left(\exp \left(\theta_{0}\right), \ldots\right.$, $\exp \left(\theta_{r}\right)$ ); angle tuples determine unique point tuples, but not vice versa. Thus we define star polygons in terms of angle tuples. This causes a certain amount of difficulty in defining the natural family of star polygons associated with a given finite set of points on the unit circle; we believe that our definition is about as simple as one can expect.

An ordered $(r+1)$-tuple of real numbers $\left(\theta_{0}, \theta_{1}, \ldots, \theta_{r}\right)$ will be called a star polygon provided $\theta_{0} \leq \theta_{1} \leq \cdots \leq \theta_{r}$ and $\theta_{r}-\theta_{0}=2 \pi d$ where $d$ is a nonnegative integer. We call $d$ the winding number. If $\theta_{i}-\theta_{i-1}=2 \pi d / r$ for each $i$, the polygon is called regular. The $r$ points (not necessarily distinct) on the unit circle given by $\exp \left(\theta_{i}\right)$, where $i=0, \cdots, r-1$, are called the vertices of the polygon, and the $r$ undirected chords $\overline{q_{i} q_{i+1}}$, where $i=0, \cdots, r-1$ and $q_{i}=$ $\exp \left(\theta_{i}\right)$ for $i=0, \cdots, r$, are called the edges of the polygon.

We now show that $n$ points $p_{0}, \cdots, p_{n-1}$ on the unit circle determine a natural family of star polygons. There is a unique angle $\theta_{i}$ in $[0,2 \pi)$ such that $\exp \left(\theta_{i}\right)=p_{i}$. By reindexing the $p_{i}$ we may assume that $\theta_{0} \leq \theta_{1} \leq \cdots \leq \theta_{n-1}$. It is convenient to think of the indices as least nonnegative residues modulo $n$. If $k$ is an integer which satisfies $1 \leq k \leq[n / 2]$ (actually the construction of this paragraph only requires $1 \leq k \leq n-1$ ), each cycle of the permutation $i \rightarrow i+k$ $(\bmod n)$ determines a star polygon as follows. Let $a_{0}$ be the least residue occurring in the cycle. Form the ordered tuple $\left(\theta_{a_{0}}, \theta_{a_{1}}, \ldots\right)=\left(\theta_{a_{0}}, \theta_{a_{0+k}}, \ldots\right.$, $\left.\theta_{n-k+a_{0}}, \theta_{a_{0}}\right)$. This set is not a star polygon; however, if we replace $\theta_{a_{j}}$ by $\theta_{a_{j}}^{\prime}=\theta_{a_{j}}+2 \beta_{j} \pi$ where $\beta_{j}$ counts the number of $i<j$ such that $a_{i+1}<a_{i}$, we obtain a star polygon.

For example, if $k=1$ we obtain the single polygon $\left(\theta_{0}, \theta_{1}, \cdots, \theta_{n-1}, \theta_{0}+\right.$ $2 \pi)$. If $k=2$ and $n$ is odd we obtain a single polygon $\left(\theta_{0}, \theta_{2}, \cdots, \theta_{n-1}, \theta_{1}+\right.$ $\left.2 \pi, \cdots, \theta_{0}+4 \pi\right)$. If $n=10$ and $k=4$ we obtain the two polygons $\left(\theta_{0}, \theta_{4}, \cdots\right.$, $\left.\theta_{2}+2 \pi, \ldots, \theta_{0}+4 \pi\right)$ and $\left(\theta_{1}, \theta_{5}, \ldots, \theta_{3}+2 \pi, . ., \theta_{1}+4 \pi\right)$. In general there will be $s=(k, n)$ polygons, each having winding number $k / s$.

If $p_{0}, \cdots, p_{n-1}$ are points on the unit circle, generate the star polygons corresponding to all integers $k$ with $1 \leq k \leq[n / 2]$. Then there is a one-to-one correspondence between the edges of the star polygons they determine and the $\left(\begin{array}{c}n \\ 2\end{array}\right)$ chords $\overline{p_{i} p_{j}}$. For example, if $n=10$ the chord $\overline{p_{2} p_{9}}$ is an edge of the polygon determined by the single cycle of the permutation $i \rightarrow i+3$. By studying certain functions defined on star polygons (the sum or product of edge lengths, for example) we obtain information about related functions defined on all $\left(\begin{array}{l}n \\ 2\end{array}\right)$ chords.

The proof of our first lemma is straightforward; we omit it. We remark that 
if a star polygon obtained from a cycle of the permutation $i \rightarrow i+k(\bmod n)$ has $r$ vertices, then $(r, d)=1$.

Lemma 2.1. Suppose $2 \theta_{i}=\theta_{i-1}+\theta_{i+1}$ for $i=1, \ldots, r-1$ and $\theta_{r}-\theta_{0}=$ $2 \pi d$. Then the star polygon $\left(\theta_{0}, \theta_{1}, \ldots, \theta_{r}\right)$ is regular and its vertices lie on the vertices of a regular $r / s$-gon where $s=(r, d)$.

Let $f=f\left(\theta_{0}, \cdots, \theta_{r}\right)$ be a real function defined on all star polygons with $r$ vertices. Let $b(r, d)$ be the value assumed by $f$ on the regular star polygon for which $\theta_{k}=2 \pi k d / r$ where $k=0, \ldots, r$.

Definition 2.1. Let $\mathcal{F}$ be the class of all those $f$, described above, which satisfy the following three conditions:

(i) $f\left(\theta_{0}+c_{0}, \cdots, \theta_{r}+c_{r}\right)=f\left(\theta_{0}, \cdots, \theta_{r}\right)$ if $c_{i} \equiv c_{j}(\bmod 2 \pi)$ for all $i, j$, and both arguments are star polygons.

(ii) $b(r, 0)<b(r, 1)<\cdots<b(r,[r / 2])$.

(iii) If $f$ is restricted to polygons with fixed winding number then $f$ achieves a maximum, and this maximum can occur only if $\theta_{i} \equiv 1 / 2\left(\theta_{i-1}+\theta_{i+1}\right)(\bmod \pi)$ for $0<i<r$.

We obtain the class $\mathcal{F}^{\prime}$ by reversing the inequalities in (ii) and replacing "maximum" by "minimum" in (iii).

In practice, (i) and (ii) are usually simple to verify. The functions $\Pi_{i=1}^{r}\left|\exp \left(\theta_{i}\right)-\exp \left(\theta_{i-1}\right)\right|$ and $\sum_{i=1}^{r}\left|\exp \left(\theta_{i}\right)-\exp \left(\theta_{i-1}\right)\right|$ are quickly seen to satisfy them. Easy compactness and convexity arguments show that (iii) is also satisfied by these functions.

Theorem 2.1. Suppose $f$ belongs to $\mathcal{F}\left(\mathcal{F}^{\prime}\right)$, and is restricted to those polygons with winding number $d \leq[r / 2]$ Then $b(r, d)$ is the maximum (minimum) value of $f$ on this class; $f$ assumes the value $b(r, d)$ only if the polygon is regular.

Proof. Suppose $d=0$. Condition (i) implies that $f\left(\theta_{0}, \theta_{1}, \cdots, \theta_{r}\right)=$ $f(0,0, \ldots, 0)=b(r, 0)$. Since any star polygon of winding number 0 is trivially regular, the theorem is true when $d=0$. Suppose the theorem is true for all $d^{\prime}<$ $d \leq[r / 2]$. Let $\left(\theta_{0}, \cdots, \theta_{r}\right)$ be a polygon of winding number $d$ on which $f \in \mathcal{F}$ achieves its maximum. For each $i$ with $1<i<r$, condition (iii) gives

$$
\theta_{i+1}-\theta_{i}=\theta_{i}-\theta_{i-1}+2 l_{i} \pi
$$

where $l_{i}$ is an integer. If $l_{i} \neq 0$, one of the differences, say $\theta_{i+1}-\theta_{i}$, is at least $2 \pi$. By (i),

$$
f\left(\theta_{0}, \ldots, \theta_{i}, \theta_{i+1}-2 \pi, \ldots, \theta_{r}-2 \pi\right)=f\left(\theta_{0}, \ldots, \theta_{r}\right) \leq b(r, d-1)
$$


This contradicts the fact that $f\left(\theta_{0}, \cdots, \theta_{r}\right) \geq b(r, d)>b(r, d-1)$. Thus $l_{i}=0$ for each $i$, and $\theta_{i+1}-\theta_{i}=2 \pi d / r$ for each $i$ by Lemma 2.1. This completes the proof.

The importance of condition (ii) on the members of $\mathcal{F}$ should not be overlooked. Suppose $f$ is the real function defined on all star polygons with $r$ vertices by $f\left(\theta_{0}, \ldots, \theta_{r}\right)=1$ if $\theta_{i}=\theta_{j}(\bmod 2 \pi)$ for each $i, j$ and $f\left(\theta_{0}, \ldots, \theta_{r}\right)=0$ otherwise. Conditions ( $i$ ) and (iii) are satisfied, but the conclusion of Theorem 2.1 does not hold.

It follows by way of the cyclic decomposition described earlier that if $n$ points lie on the circumference of the unit circle, then both the product and sum of the lengths of the $\left(\begin{array}{l}n \\ 2\end{array}\right)$ chords they determine are maximal when the points are the vertices of a regular $n$-gon. To prove this, apply Theorem 2.1 to the two distance functions mentioned earlier.

To put this result in the language of the introduction, let $K$ be the unit circle, $f(x, y)=|x-y|$ or $\log |x-y|$, and let $\pi$ be the $n$-discrete measures on $K$. Then $I(K)=n^{-1} \cot (\pi / 2 n)$ or $(2 n)^{-1} \log n$ respectively (see Fejes Tóth [7] and Schur $[30]$ respectively).

The following corollary generalizes the results on the sum and product of chord lengths. Since there are no new ideas involved, aside from elementary technical details, we omit the proof.

Corollary 2.1. Let $g(x)=e^{-a x} x^{b}(\log x)^{c}$, and

$$
r(a, b, c)= \begin{cases}(-1)^{c} & \text { for } a \geq 0, b \leq 0, c \text { a positive integer, } \\ 1 & \text { for } a \geq 0, b \leq 0, c=0, a^{2}+b^{2} \neq 0, \\ -1 & \text { for } a=0,0<b \leq 1, c=0 .\end{cases}
$$

Let $s_{i j}=\left|\exp \left(\theta_{i}\right)-\exp \left(\theta_{j}\right)\right|$. Then $\Sigma_{i<j} g\left(1 / 2 s_{i j}\right)$ is maximal (minimal) if and only if the points $p_{i}=\exp \left(\theta_{i}\right)$ are the distinct vertices of a regular n-gon, provided $\tau=-1(+1)$.

We remark that the result on the product of chord lengths comes by setting $a=b=0$, and $c=1$. Also, if $0<\lambda \leq 1$, we may set $a=c=0, b=\lambda$ to see that $\Sigma_{i, j} s_{i j}^{\lambda}$ maximizes for the regular $n$-gon. If $\lambda>1$, the methods of this section fail because we no longer have the required convexity. Condition (iii) is not satisfied. Later on, we will deal with $1<\lambda<2$. The case $\lambda=2$ is easily treated as follows.

Suppose $p_{1}, p_{2}, \cdots, p_{n}$ are unit vectors in an inner product space. We note the identity

$$
\sum_{i<j}\left|p_{i}-p_{j}\right|^{2}=n^{2}-\left|\sum_{i} p_{i}\right|^{2} .
$$


The identity shows that if $n$ points lie on a unit sphere, the sum of the squares of the various distances they determine is at most $n^{2}$, and will be $n^{2}$ if and only if the centroid of the points is the origin. Thus a regular $n$-gon represents a maximal configuration which will not be unique for $n>3$. For $\lambda>2$, the sum $\Sigma_{s} \lambda$ does not generally maximize on a regular n-gon. The paper of Björck [2] gives a nice treatment of this problem when $\lambda>2$.

In the final theorem of this section we give another generalization of the results about sums and products of chord lengths.

Theorem 2.2. Let $\sigma$ be an elementary symmetric function on $\left(\begin{array}{c}n \\ 2\end{array}\right)$ real variables. Let $\left\{s_{i j}\right\}$ be the lengths of the chords determined by $n$ points on the circumference of the unit circle. Then $\sigma\left(s_{i j}\right)$ is maximal if and only if the $n$ points are the distinct vertices of a regular n-gon.

We will need the following lemma.

Lemma 2.2. Let $\mu$ be an elementary symmetric function on $r$ real variables. Let $s_{1}, \cdots, s_{r}$ denote the lengths of the edges of a star polygon $T=\left(\theta_{0}, \ldots, \theta_{r}\right)$ Then $\bar{\mu}\left(\theta_{0}, \cdots, \theta_{r}\right)=\mu\left(s_{1}, \cdots, s_{r}\right)$ belongs to the class $\mathcal{F}$.

Proof. It is clear that $\bar{\mu}$ satisfies conditions (i) and (ii). Suppose $T$ has winding number $d \leq[r / 2]$. A standard compactness argument shows that a configuration exists for which $\vec{\mu}\left(\theta_{0}, \ldots, \theta_{r}\right)$ is maximal. Let $s_{i}$ be the length of the edge $\overline{p_{i-1} p_{i}}$. We may write

$$
\bar{\mu}\left(\theta_{0}, \ldots, \theta_{r}\right)=s_{i} s_{i+1} A+\left(s_{i}+s_{i+1}\right) B+C
$$

where $A, B$ and $C$ are numbers which are independent of $s_{i}$ and $s_{i+1}$. If $\theta_{i} \not \equiv$ $1 / 2\left(\theta_{i-1}+\theta_{i+1}\right)(\bmod \pi)$, then the point $p_{i}$ may be moved to increase both $s_{i} s_{i+1}$ and $s_{i}+s_{i+1}$, while leaving the winding number unchanged. This shows that $\bar{\mu}$ satisfies condition (iii).

From Lemma 2.1 and Theorem 2.1 it follows that $\bar{\mu}$, when restricted to star polygons with winding number $d$, is maximal when $\theta_{i+1}-\theta_{i}=2 \pi d / r$.

Proof of Theorem 2.2. Suppose $\sigma$ is the lth symmetric function; $\sigma\left(s_{i j}\right)$ will be the sum of all $l$-fold products of chord lengths determined by the points $p_{0}$, $\cdots, p_{n-1}$. Let $T_{1}, \cdots, T_{t}$ be all of the star polygons obtained by the decomposition described at the beginning of this section. Each $l$-fold product $\beta$ can be written uniquely as $\beta=q_{1} q_{2} \cdots q_{t}$ where $q_{i}$ is a product of lengths of edges occurring in $T_{i}$ (If there are no edges from $T_{i}$, set $q_{i}=1$.) Associated with each product $\beta$ is a set of occupancy numbers $k_{1}, k_{2}, \cdots, k_{t}$ with $\Sigma_{i} k_{i}=l$, where $k_{i}$ is the number of edge lengths from $T_{i}$ which occur in the product $q_{i}$.

We group together all $l$-fold products which have exactly the same set of 
occupancy numbers $k_{1}, \cdots, k_{t}$. The sum of this collection of products can be written as

$$
\Pi\left(k_{1}, \ldots, k_{t}\right)=\mu_{1}\left(k_{1}\right) \mu_{2}\left(k_{2}\right) \ldots \mu_{t}\left(k_{t}\right)
$$

where $\mu_{i}\left(k_{i}\right)$ is the $k_{i}$ th symmetric function on the set of edge lengths determined by $T_{i}$. We define $\mu_{i}(0)=1$.

Since each $\mu_{i}\left(k_{i}\right)$ is maximal when $T_{i}$ is a regular star polygon, we immediately deduce that $\Pi\left(k_{1}, \cdots, k_{t}\right)$ is maximal when the points $\left\{p_{i}\right\}$ are the distinct vertices of a regular $n$-gon. If $T_{1}$ is the star polygon with $n$ sides and winding number $1, \mu_{1}(k)$ is maximal only when the $\left\{p_{i}\right\}$ determine a regular n-gon. It follows that $o\left(s_{i j}\right)$ is maximal only for this configuration by summing over all the various sets of occupancy numbers. This completes the proof.

We can now sketch results for the non-Euclidean case. Say two points $\boldsymbol{p}_{\boldsymbol{i}}, \boldsymbol{p}_{j}$ on a non-Euclidean circle $C_{\rho}$ of radius $\rho$ determine a central angle of $\theta, 0 \leq \theta$ $\leq \pi$. Let $0<r \leq R$. Then the distance between them is

$$
\begin{aligned}
d\left(p_{i}, p_{j}\right) & =R \operatorname{arctanh}\left[\frac{2 r}{R} \frac{(\sin 1 / 2 \theta)}{\left(1-2 r^{2} R^{-2} \cos \theta+r^{4} R^{-4}\right)^{1 / 2}}\right], \quad \rho=R \operatorname{arctanh} r / R, \\
& =\operatorname{arctanh}\left[\frac{(2 r \sin 1 / 2 \theta)\left(R^{2}-r^{2} \cos ^{2} 1 / 2 \theta\right)^{1 / 2}}{\left(R^{2}-r^{2} \cos \theta\right)}\right], \quad \rho=\frac{\operatorname{arctanh} r / R}{\operatorname{arctanh} 1 / R}, \\
& =2 r \sin 1 / 2 \theta, \quad \rho=r, \\
& =2 R \arcsin \left(r R^{-1} \sin 1 / 2 \theta\right), \quad \rho=R \arcsin r / R .
\end{aligned}
$$

depending upon whether the circle is Lobatchevskian (in the conformal model), Lobatchevskian with curvature arc tanh $1 / R$, Euclidean, or Riemannian with curvature $1 / R$.

In each case elementary calculus shows that $f(\theta)=d\left(p_{i}, p_{j}\right)$ is positive, strictly increasing for $0 \leq \theta \leq \pi$, and concave in $\theta$. It follows that $d^{\lambda}\left(p_{i}, p_{j}\right)$ and $\log d\left(p_{i}, p_{j}\right)$ are also concave in $\theta$. Thus the same ideas as before yield

Theorem 2.2' . Let $p_{0}, \cdots, p_{n-1}$ be $n$ points on a non-Euclidean circle and let $d\left(p_{i}, p_{j}\right)$ be the non-Euclidean distance between $p_{i}$ and $p_{j}$. Let $\sigma$ be an elementary symmetric function on $\left(\begin{array}{l}n \\ 2\end{array}\right)$ real variables. Then for $0<\lambda \leq 1$, $\sigma\left(d^{\lambda}\left(\phi_{i}, p_{j}\right)\right)$ is maximal only on a regular n-gon.

Handy references for distance formulas in non-Euclidean spaces are [4, pp. 16-21] and [11, pp. 237-241].

It is a consequence of Theorem $2.2^{\prime}$ that for points $p_{0}, \cdots, p_{n-1}$ on $C_{p}$ 


$$
\begin{aligned}
& \lim _{n \rightarrow \infty} \frac{1}{n^{2}} \max \sum_{0 \leq i \leq j \leq n-1} d\left(p_{i}, p_{j}\right) \\
& =\frac{2 \rho}{\pi} \cdot \frac{1}{(\rho \kappa)} \int_{0}^{\tanh (\rho \kappa)} \frac{\arcsin t}{t\left(1-t^{2}\right)} d t, \quad \kappa=\operatorname{arctanh} 1 / R, \\
& =2 \rho / \pi, \quad \kappa=0, \\
& =\frac{2 \rho}{\pi} \cdot \frac{1}{(\rho \kappa)} \int_{0}^{\sin (\rho \kappa) \operatorname{arctanh} t} \frac{\tan }{t} d t, \quad \kappa=1 / R,
\end{aligned}
$$

for the last three geometries, where $\kappa$ is the curvature of the corresponding space. Since the maximum occurs at the regular $n$-gon, we simply use the fact that the Riemann sums of a continuous function tend to its integral. The details are omitted.

If $(\rho \kappa)^{3}$ is negligible then the values are respectively

$$
\frac{2 \rho}{\pi}\left[1+\frac{1}{18}(\rho \kappa)^{2}\right], \quad \frac{2 \rho}{\pi}, \quad \frac{2 \rho}{\pi}\left[1-\frac{1}{18}(\rho \kappa)^{2}\right] .
$$

In fact these expressions are the same aside from \pm signs, since $-i$ arc $\sin (i \tan x)=\operatorname{arctanh}(\sin x)$. The integrals can be expressed in terms of Lobatchevsky's function $L(x)=-\int_{0}^{x} \log \cos t d t$.

We close this section with two unanswered questions. Let $K$ be the unit circle, $f(x, y)=|x-y|^{\lambda}$ with $1<\lambda<2$, and $\mathbb{N}$ the $n$-discrete measures. Is the extremal measure $\mu_{0}$ given by the vertices of the regular $n$-gon, as is the case for $0<\lambda \leq 1$ ? Let $K$ be the unit circle, $f(x, y)=|x-y|$, and $\pi$ those measures consisting of $n$ positive atoms $a_{1}, \cdots, \alpha_{n}$. Is the $n$-discrete measure determined by the vertices of a regular $n$-gon extremal?

3. Application of metric embedding theory. Let $K$ consist of $n$ distinct points $p_{1}, \cdots, p_{n}$ in a Euclidean space and $M$ consist of all signed measures of total mass one supported by $K$. In this section we will investigate and interpret the number $I(K)$ when the kernel function $f(x, y)$ is $|x-y|^{\lambda}$, and $0<\lambda \leq 2$. We will apply our results to several related problems.

For each $\mu$ in $\mathbb{N}$, we have $I(K, \mu)=\Sigma_{i j}\left|p_{i}-p_{j}\right|^{\lambda} x_{i} x_{j}$ where $\Sigma_{i} x_{i}=1$ and $\mu\left(p_{i}\right)=x_{i}$. The following two theorems of I. J. Schoenberg [26] give us the information we need.

Theorem 3.1 (Schoenberg). Let $p_{1}, \cdots, p_{n}$ be $n$ distinct points in a $E u$ clidean space. Let $s_{i j}$ be the distance between $p_{i}$ and $p_{j}$. The quadratic form 
$\Sigma_{i j} s_{i j}^{\lambda} x_{i} x_{j}$ is nonsingular, and is negative definite on the byperplane $\Sigma_{i} x_{i}=0$, provided $0 \leq \lambda<2$.

The following corollary follows at once from simple considerations of continuity.

Corollary 3.1. Let $p_{1}, \cdots, p_{n}$ be $n$ points, not necessarily distinct, in a Euclidean space. Then the quadratic form $\Sigma_{i j} s_{i j}^{\lambda} x_{i} x_{j}$ is negative semidefinite on the byperplane $\Sigma x_{i}=0$, provided $0<\lambda \leq 2$.

Theorem 3.2 (Schoenberg). The symmetric $(n+1) \times(n+1)$ matrix

$$
M=\left(\begin{array}{ll}
0 & 1 \\
1 & s_{i j}^{\lambda}
\end{array}\right)
$$

is nonsingular for $0 \leq \lambda<2$ provided that $p_{1}, \cdots, p_{n}$ are distinct points in a Euclidean space.

We can now easily prove the following theorem.

Theorem 3.3. Let $p_{1}, \cdots, p_{n}$ be distinct points in a Euclidean space. The quadratic form $\Sigma_{i j} s_{i j}^{\lambda} x_{i} x_{j}$ acbieves a unique absolute maximum on the byperplane $\Sigma x_{i}=1$, provided $0 \leq \lambda<2$. The maximum point will be determined by the unique solution of the following system of $(n+1)$ equations in $(n+1)$ unknowns:

$$
\sum_{i=1}^{n} x_{i}=1, \quad x_{0}+\sum_{i=1}^{n} s_{i j}^{\lambda} x_{i}=0, \quad j=1,2, \ldots, n .
$$

The unknown $x_{0}$ is a Lagrange multiplier.

Proof. By Theorem 3.1 the form is negative definite on the hyperplane $\Sigma x_{i}=$ 0 , and hence will achieve a maximum on any parallel hyperplane such as $\Sigma x_{i}=1$. We apply the method of Lagrange to

$$
f\left(x_{0}, \ldots, x_{n}\right)=x_{0}\left(\sum_{i=1}^{n} x_{i}\right)+\sum_{i j} s_{i j}^{\lambda_{i j} x_{i} x_{j}}
$$

We see that at a maximum, the system of equations (3.1) must be satisfied. The matrix of the system is precisely the $M$ of Theorem 3.2. Since $M$ is nonsingular, the system possesses a unique solution. This completes the proof.

Corollary 3.2. If $x_{i}=m_{i} / m$, where $m_{i}$ is a nonnegative integer for $i=1,2$, $\cdots, n$, is a solution of the system (3.1), then $x_{i}=m_{i} / m$ must give the solution to the problem of finding an extremal m-discrete measure on $K$. 
Lemma 3.1. Let $K$ be a compact convex polytope in Euclidean space. Let $n$ variable points $p_{1}, \cdots, p_{n}$ lie in $K$. Then if $\lambda \geq 1, \Sigma_{i j}^{\lambda}$ will acbieve a maximal value for a configuration in which each $p_{i}$ is a vertex of $K$. Thus an extremal $n$-discrete measure on $K$ is supported by the vertices.

Proof. The proof is a straightforward application of standard compactness and convexity arguments. We remark that except when $\lambda=1$, and $K$ is a line segment, the maximal value can occur only if each $p_{i}$ is a vertex of $K$.

Definition 3.1. A set of points $p_{1}, \cdots, p_{n}$ in a Euclidean space will be called $\lambda$-homogeneous if for each $j$, the sum $\Sigma_{i=1}^{n} s_{i j}^{\lambda}$ is a constant independent of $j$. A convex polytope will be called $\lambda$-homogeneous if its vertices form a $\lambda$-homogeneous set.

Regular polygons, the Platonic solids, rectangles of all dimensions, etc., are $\lambda$-homogeneous for all $\lambda$. The term "strictly metrically homogeneous" has been used by Grünbaum and Kelly [0] to denote a set of points for which the set (counting multiplicities) of distances $\left\{s_{1 j}, \ldots, s_{n j}\right\}$ is independent of $j$. Clearly, "strictly homogeneous" is equivalent to " $\lambda$-homogeneous for all $\lambda$ ". However, it is easy to find 1-homogeneous sets which are not strictly homogeneous, etc.

Theorem 3.4. Suppose $1 \leq \lambda<2$. Let $K$ be a convex $\lambda$-bomogeneous polytope with $n$ vertices and let $f(x, y)=|x-y|^{\lambda}$. Then the unique extremal $n$ discrete measure is obtained by placing an atom at eacb vertex.

Proof. By Lemma 3.1 an extremal measure will occur only when each atom is at some vertex of $K$. We observe that $x_{i}=1 / n, i=1, \cdots, n$, and $x_{0}=$ $-n^{-1}\left(\sum_{i=1}^{n} s_{i j}^{\lambda}\right)$ is a solution of the system (3.1). Hence this must be the unique solution. If the atoms are constrained to lie on vertices of $K$, then the result is true for $\lambda$ in the range $0 \leq \lambda<2$.

For example, if twelve points are placed in an icosahedron, the sum of the sixty-six distances they determine will be uniquely maximal when each point is a distinct vertex. Moreover, if $K$ is any polytope with a nontrivial symmetry group, it is clear that whenever two vertices belong to the same orbit, then the corresponding $x_{i}$ in the solution vector of (3.1) are equal.

If $K$ is a regular $n$-gon, $f(x, y)=|x-y|^{\lambda}$ with $1 \leq \lambda<2$, and $\pi$ consists of measures made of $n$ nonnegative atoms, then the method of Theorem 3.4 shows that the extremal measure is obtained by placing an atom of weight $1 / n$ at each vertex. (Compare with the second question at the end of $\$ 2$.)

If $K$ is not $\lambda$-homogeneous, it generally happens that the solution vector of (3.1) contains $x_{i}$ which are negative. We now give a geometrical interpretation of the solution vector of (3.1) which sheds considerable light. The following theorem 
follows from the paper of Schoenberg [26]. We let $R$ denote the set of real numbers.

Theorem 3.5 (Schoenberg). If $d$ is the Euclidean metric, then the metric space $\left(R^{m}, d^{\sigma}\right), 0<\sigma \leq 1$, can be isometrically embedded in the sequence space $l_{2}$. Moreover, if $\sigma<1$ and $p_{0}, p_{1}, \cdots, p_{n}$ are distinct points in $E^{m}=\left(R^{m}, d\right)$, their images $p_{0}^{\prime}, \cdots, p_{n}^{\prime}$ in $\left(R^{m}, d^{\sigma}\right)$ considered as a subset of $l_{2}$ form the vertices of a nondegenerate n-simplex.

Lemma 3.2. Let $p_{0}, \cdots, p_{n}$ form the vertices of a nondegenerate n-simplex in $E^{n}$. Then the maximum value assumed on the byperplane $\Sigma_{i} x_{i}=1$ by the form $\Sigma_{i<j} s_{i j}^{2} x_{i} x_{j}$ is $\rho^{2}$, where $\rho$ is the radius of the circumsphere of $p_{0}, \cdots, p_{n}$.

Proof. Let the origin be the center of the circumsphere. Now

$$
\begin{gathered}
2\left(\sum_{i<j} s_{i j}^{2} x_{i} x_{j}\right)=\sum_{i, j}\left[\left(p_{i}-p_{j}\right) \cdot\left(p_{i}-p_{j}\right)\right] x_{i} x_{j} \\
\quad=\sum_{i, j}\left(\left|p_{i}\right|^{2}+\left|p_{j}\right|^{2}-2 p_{i} \cdot p_{j}\right) x_{i} x_{j} \\
\quad=2\left[\rho^{2}\left(\sum_{i} x_{i}\right)^{2}-\sum_{i, j}\left(x_{i} p_{i} \cdot x_{j} p_{j}\right)\right] .
\end{gathered}
$$

Thus

$$
\sum_{i<j} s_{i j}^{2} x_{i} x_{j}=\rho^{2}-\left|\sum_{i} x_{i} p_{i}\right|^{2}
$$

There will be a unique choice of $x_{0}, \cdots, x_{n}$ for which $\Sigma_{i} x_{i} p_{i}=0$.

We also see that the $x_{i}$ are all nonnegative only if the center of the circumsphere lies in the convex hull of the $p_{i}$. In general, the solution vector is given by the affine (barycentric) coordinates of the center of the circumsphere.

Theorem 3.6. Let $p_{0}, \cdots, p_{n}$ be points in $E^{m}$ which form a $\lambda$-bomogeneous set. Then the points $p_{0}^{\prime}, \cdots, p_{n}^{0}$ in $\left(R^{m}, d^{\lambda / 2}\right) \subset l_{2}$ bave their centroid at the center of their circumsphere. Moreover, if the set is strictly bomogeneous, then $p_{0}, \cdots, p_{n}$ lie on a sphere in $E^{m}$ with their centroid as its center.

Proof. Introduce variables $x_{i}$ as in Lemma 3.2. The equations corresponding to (3.1) are

$$
\sum_{i=0}^{n} x_{i}=1, \quad x_{-1}+\sum_{i=0}^{n} s_{i j}^{\lambda} \dot{x}_{i}=0, \quad j=0,1, \ldots, n,
$$


where $x_{-1}$ is the Lagrange multiplier. If $p_{0}, \cdots, p_{n}$ are $\lambda$-homogeneous, where $0<\lambda<2$, then the solution to this system. of equations is

$$
x_{i}=\frac{1}{(n+1)}, i=0, \ldots, n ; \quad x_{-1}=-\frac{1}{(n+1)} \sum_{i=0}^{n} s_{i j}^{\lambda} .
$$

Formula (3.2) implies that if $p_{0}^{\prime}, \cdots, p_{n}^{\prime}$ are the images in $E^{n} \subset l_{2}$ given by Theorem 3.5, then their centroid is the center of their circumsphere. If the set is strictly homogeneous, we may use a continuity argument as $\lambda \rightarrow 2$. Let us assume that $E^{m}$ is the Euclidean space of least dimension containing $p_{0}, \cdots, p_{n}$; thus $E^{m} \subset E^{n}$. Let $p_{0}^{\prime}, \cdots, p_{m}^{\prime}$ lie on a sphere of radius $\rho_{\lambda}$ centered at the origin of $E^{n}$. As $\lambda \rightarrow 2$, these spheres converge to a sphere of radius $\rho=$ $(n+1)^{-1}\left(\Sigma_{1<j} s_{i j}^{2}\right)^{1 / 2}$ which (by continuity) will contain an isometric copy of $p_{0}, \cdots, p_{n}$.

When $m=2$, this result on strictly homogeneous sets follows from their characterization by Grünbaum and Kelly [0]. Perhaps Theorem 3.6 is a step towards deciding whether or not a strictly homogeneous set does possess a nontrivial symmetry group.

The question has a negative answer if there exists a Latin square with the following properties: the square is constant down the main diagonal, is symmetric with respect to this main diagonal, and has a trivial group of automorphisms. By "automorphism" we mean a symmetric row and column permutation which gives a square identical with the original. If the square is $(n+1) \times(n+1)$, replace the diagonal letter by zero and the other letters by $1, \ldots, n$ to obtain a matrix $\left(a_{i j}\right)$. For $\lambda>0$ sufficiently small, the work of Schoenberg assures us that $\left(a_{i j}^{\lambda}\right)$ represents the distance matrix for a set of points in $E^{n}$. The symmetry group would be trivial.

Next let $K$ be a compact set in a Euclidean space, and let $\mu$ be a signed Borel measure on $K$ with total mass one. As usual let $I(K, \mu)=$ $\iint|p-q| d \mu(p) d \mu(q)$ and $I(K)=\sup _{\mu} I(K, \mu)$. Björck [2] has made a thorough study of $I(K, \mu)$ in the case where $\mu$ is a positive Borel measure. Let $I^{+}(K)$ be the supremum of $I(K, \mu)$ taken over positive $\mu$.

Theorem 3.7 (Björck). Let $K$ be a compact set in $E^{m}$. Then there is a unique positive Borel measure $\mu$ such that $I(K, \mu)=I^{+}(K)$.

We will prove the following result about $I(K)$.

Theorem 3.8. Let $K$ be a compact set in $E^{m}$. The metric space $\left(K, d^{1 / 2}\right)$ may be isometrically embedded on the surface of a Hilbert sphere of radius $\rho<$ $a(m)$. Diameter $(K)$. Moreover, $I(K)=2 \bar{\rho}^{2}<\propto$ where $\bar{\rho}$ is the least sucb $\rho$.

The proof will use Lemmas 3.3-3.5; we abbreviate Diameter $(K)$ by $D(K)$. 
Lemma 3.3. We bave $I(K)=\sup I(K, \mu)$ where $\mu$ is concentrated on finitely many atoms.

Proof. The kernel function $|p-q|$ is uniformly continuous on $K \times K$. Decompose $K$ into disjoint Borel sets $K_{1}, \cdots, K_{s}$ such that for all $i, j$ the products $K_{i} \times K_{j}$ have diameters so small that the variation of $|p-q|$ is less than $\epsilon$ on $K_{i} \times K_{j}$. Let $p_{i}$ be in $K_{i}$ and define $\bar{\mu}\left(p_{i}\right)=\mu\left(K_{i}\right)$. Since $I(K, \bar{\mu})=$ $\iint g(p, q) d \mu(p) d \mu(q)$ where $g(p, q)=\left|p_{i}-p_{j}\right|$ for $(p, q)$ in $K_{i} \times K_{j}$, we see that $|I(K, \mu)-I(K, \bar{\mu})| \leq \epsilon|\mu \times \mu|(K \times K)$.

Lemma 3.4 (Menger). A separable metric space $(M, \delta)$ can be isometrically embedded on the surface of a Hilbert sphere of radius $\rho$ if each finite collection of points in $(M, \delta)$ can be embedded on a Euclidean sphere of radius not exceeding $\rho$.

We suppress the standard argument. Chapters IV and V of Blumenthal's book [4] thoroughly discuss results of this nature.

Let $K^{\prime}$ be a finite collection of points in $K$. Theorem 3.5 and Lemma 3.2 tell us that $I\left(K^{\prime}\right)=2 \rho^{\prime 2}$ where $\rho^{\prime}$ is the radius of the circumsphere of $\left(K^{\prime}, d^{1 / 2}\right)$ $C l_{2}$. Thus if $I\left(K^{\prime}\right) \leq 2 \rho^{2}$ for all such $K^{\prime}$, Lemma 3.4 allows us to say that $\left(K^{\prime}, d^{1 / 2}\right)$ lies on a sphere of radius $\rho$ in $l_{2}$. Since $I(K) \geq I\left(K^{\prime}\right)$, the least such $\rho$ is $[1 / 2 I(K)]^{1 / 2}$.

The results of the above paragraph would be of little interest if $I(K)=+\infty$. We show this is not the situation.

Lemma 3.5. The metric space $\left([0,1], d^{1 / 2}\right)$ can be embedded on the Hilbert sphere of radius $1 / 2$. Since this radius is clearly minimal, $I([0,1])=1 / 2$.

Proof. Let $0=p_{0}<p_{1}<\cdots<p_{n}=1$. Consider the mapping given by $p_{i}^{\prime}=$

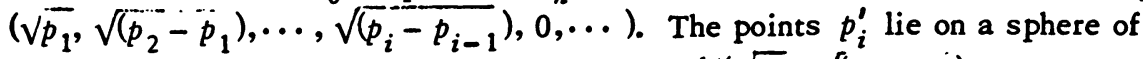
radius $1 / 2$ in $l_{2}$. The center of the sphere is $1 / 2\left(\sqrt{p_{1}}, \sqrt{\left(p_{2}-p_{1}\right), \ldots,}\right.$ $\left.\sqrt{ }\left(p_{n}-p_{n-1}\right), 0, \ldots\right)$. Hence the result follows from Lemma 3.4. By a dilation we also obtain $I([a, b])=1 / 2(b-a)$. Placing atoms of weight $1 / 2$ at $a$ and $b$ gives the extremal measure. A direct proof can be given, but we know of no neat one.

To complete the proof of Theorem 3.8 let $K^{\prime}=\left\{p_{1}, \cdots, p_{n}\right\}$ be a finite set of points in $K$. Then

$$
\begin{aligned}
\sum_{i, j}\left|p_{i}-p_{j}\right| x_{i} x_{j} & =\sum_{i, j}\left(b(m) \int\left|\left(p_{i}-p_{j}\right) \cdot t\right| d \sigma(t)\right) x_{i} x_{j} \\
& =b(m) \int\left(\sum_{i, j}\left|\left(p_{i}-p_{j}\right) \cdot t\right| x_{i} x_{j}\right) d \sigma(t) .
\end{aligned}
$$


The right side of (3.3) comes from noting that if $p, q$ are points in $E^{m}$, then $|p-q|=b(m) \int|(p-q) \cdot t| d \sigma(t)$ where the integral is over the surface of the unit $m$-ball. The sum inside the integral may be viewed as the one dimensional case by projecting the points onto a line having the direction $t$. Hence by Lemma 3.5, the inner sum is at most $1 / 2 D(K)$ if $\Sigma_{i} x_{i}=1$; thus the left side of (3.3) is at most $1 / 2 b(m) \sigma D(K)$ on this hyperplane. Hence $I(K) \leq a(m) D(K)$.

Several interesting questions remain. First, does there always exist a $\mu$ such that $I(K)=I(K, \mu)$ ? The method used here can be extended to the kernel $|p-q|^{\lambda}$ if $0<\lambda \leq 1$. What about $1<\lambda<2$ ?

We remark that we can give a geometric interpretation to $I^{+}$; we have $I^{+}(K)=$ $2\left(\rho^{2}-s^{2}\right)$ where $I(K)=2 \rho^{2}$ and $s$ is the distance to the convex hull of $\left(K, d^{1 / 2}\right)$ from the center of the Hilbert sphere of radius $\rho$ on which it is embedded by Theorem 3.8.

4. Definite semimetrics on Euclidean spheres. To define semimetric one drops the requirement that the triangle inequality be satisfied. We will be concerned only with continuous semimetrics which are invariant under Euclidean motions.

Definition 4.1. A semimetric $b$ will be called definite if the quadratic form $\Sigma_{i<i} b\left(p_{i}, p_{j}\right) x_{i} x_{j}$ is negative definite on the hyperplane $\Sigma_{x_{i}}=0$ whenever $p_{1}, p_{2}$, $\cdots, p_{n}$ are distinct points. (If the form is allowed to be negative semidefinite, we call $b$ semidefinite.)

Lemma 4.1. Let $b$ be definite, and suppose $p_{1}, \cdots, p_{n}$ and $q_{1}, \cdots, q_{n}$ are two sets of points. We bave

$$
\sum_{i<j} b\left(p_{i}, p_{j}\right)+\sum_{i<j} b\left(q_{i}, q_{j}\right) \leq \sum_{i, j} b\left(p_{i}, q_{j}\right)
$$

Equality can occur only if the sets are identical.

Proof. We consider the form

$$
\sum_{i<j} b\left(p_{i}, p_{j}\right) x_{i} x_{j}+\sum_{i<j} b\left(q_{i}, q_{j}\right) y_{i} y_{j}+\sum_{i, j} b\left(p_{i}, q_{j}\right) x_{i} y_{j}
$$

which is negative semidefinite if $\Sigma x_{i}+\Sigma y_{i}=0$. If we set each $x_{i}=1$ and each $y_{i}=-1$, the inequality is immediate. Let $0_{1}, \ldots, 0_{k}$ be the distinct points among the $p_{i}$ and $q_{i}$. Let $0_{i}$ occur with multiplicity $z_{i}^{\prime}, z_{i}^{\prime \prime}$ among the $p_{i}, q_{i}$, respectively. If $z_{i}=z_{i}^{\prime}-z_{i}^{\prime \prime}$, then $\Sigma_{i<j} b\left(0_{i}, 0_{j}\right) z_{i} z_{j}$ is negative unless $z_{i}^{\prime}=z_{i}^{\prime \prime}$ for each $i$.

If $b$ is a definite semimetric on a unit $m$-sphere, put $S(b, n)=$ $\max \left\{\Sigma_{i<j} b\left(p_{i}, p_{j}\right)\right\}$ as $p_{1}, \cdots, p_{n}$ vary over the sphere. 
Lemma 4.2. We bave $S(b, 2 n)>4 S(b, n)$.

Proof. Let $p_{1}, \cdots, p_{n}$ be such that $\Sigma_{i<j} b\left(p_{i}, p_{j}\right)=s(b, n)$. Let $q_{1}, \cdots, q_{n}$ be a nonidentical isometric copy of $p_{1}, \cdots, p_{n}$. We have

$$
s(b, 2 n) \geq \sum_{i<j}^{-} b\left(p_{i}, p_{j}\right)+\sum_{i<j} b\left(q_{i}, q_{j}\right)+\sum_{i, j} b\left(p_{i}, q_{j}\right) .
$$

By Lemma 4.1 the third sum exceeds the sum of the first and second. The result follows.

Theorem 4.1. Let $p_{0}$ be a fixed point on the m-sphere and define $c(b)$ to be $(2 \sigma)^{-1} \int b\left(p_{0}, p\right) d \sigma(p)$ where $\sigma$ is the surface content of the m-sphere. Then

$$
c(b) n(n-1)<S(b, n)<c(b) n^{2} .
$$

Proof. Let $p_{1}, \cdots, p_{n}$ be $n$ points such that $\Sigma_{i<j} b\left(p_{i}, p_{j}\right)=S(b, n)$. Let $q_{1}, \cdots, q_{k n}$ be $k n$ variable points. We always have

$$
k^{2} s(b, n)+\sum_{i<j} b\left(q_{i}, q_{j}\right) \leq k \sum_{i, j} b\left(p_{i}, p_{j}\right)
$$

The inequality (4.3) is obtained by setting $x_{i}=k$ and $y_{i}=-1$ in the proof of Lemma 4.1. For"convenience write (4.3) in the form $\Sigma_{1}+\Sigma_{2} \leq \Sigma_{3}$. We note that

(i) $\sigma^{-k n} \int \cdots \int \Sigma_{1} d \sigma\left(q_{1}\right) \cdots d \sigma\left(q_{k n}\right)=k^{2} S(b, n)$,

(ii) $\sigma^{-k n} \int \cdots \int \Sigma_{2} d \sigma\left(q_{1}\right) \cdots d \sigma\left(q_{k n}\right)=k n(k n-1) c(b)$, and

(iii) $\sigma^{-k n} \int \ldots \int \Sigma_{3} d \sigma\left(q_{1}\right) \ldots d \sigma\left(q_{k n}\right)=2 k^{2} n^{2} c(b)$.

The inequality (4.3) implies that $k^{2} S(b, n)+k n(k n-1) c(b) \leq 2 k^{2} n^{2} c(b)$; thus

$$
s(b, n) \leq\left(n^{2}+n / k\right)_{c}(b) \text {. }
$$

Letting $k$ tend to infinity gives $S(b, n) \leq n^{2} c(b)$. If equality were to hold, Lemma 3.2 would give $S(b, 2 n)>c(b)(2 n)^{2}$, a contradiction.

If $p_{1}, \cdots, p_{n}$ are variable points on the $m$-sphere, then

$$
s(b, n)>\sigma^{-n} \int \cdots \int \sum_{i<j} b\left(p_{i}, p_{j}\right) d \sigma\left(p_{1}\right) \cdots d \sigma\left(p_{n}\right) .
$$

The left inequality in (4.2) follows.

Fejes Tóth [7] conjectured that if $n$ points $p_{1}, \cdots, p_{n}$ are distributed on the 2-sphere then $\Sigma_{i<j} d\left(p_{i}, p_{j}\right)<2 n^{2} / 3$. The previously cited work of Schoenberg assures us that $d$ is definite, and an easy computation shows $c(d)=2 / 3$ for the 2-sphere. Actually, this result is implicit in the paper of Björck [2], and the potential theoretic methods he uses can certainly be developed to obtain the right inequality in (4.2). However, refinements of the methods used here are proving 
useful in obtaining error estimates for the interesting $n$-discrete situation; see [1], [32], and [33].

Next we wish to show that the great circle metric $d^{\prime}$ is semidefinite. Let $p_{1}, p_{2}, \cdots, p_{n+1}, \cdots, p_{2 n}$ be points on a unit circle, indexed in counterclockwise order, such that $p_{i}$ and $p_{i+n}$ are antipodal. On the hyperplane $\Sigma_{i} x_{i}=0$ we have

$$
\begin{aligned}
& -\sum_{i<j} d^{\prime}\left(p_{i}, p_{j}\right) x_{i} x_{j}=s_{1}\left(x_{2}+\cdots+x_{n+1}\right)^{2} \\
& \quad+s_{2}\left(x_{3}+\cdots+x_{n+2}\right)^{2}+\cdots+s_{n}\left(x_{n+1}+\cdots+x_{2 n}\right)^{2} .
\end{aligned}
$$

Here $s_{i}=d^{\prime}\left(p_{i}, p_{i+1}\right)=d^{\prime}\left(p_{i+n}, p_{i+n+1}\right)$. To verify (4.6), set $x_{i}=1, x_{j}=-1$, and the other variables equal to zero. Professor Richard Bishop has pointed out the following "differentiable" analogue of (4.6); if $f$ has period $2 \pi$ then

$$
-\int_{-\pi}^{\pi} \int_{y-\pi}^{y+\pi}|x-y| f^{\prime}(x) f^{\prime}(y) d x d y=\int_{-\pi}^{\pi}[f(y)-f(y-\pi)]^{2} d y .
$$

The mass distribution is given by $f^{\prime}$. Equations (4.6) and $\left(4.6^{\prime}\right)$ are special cases of a general Stieltjes integral formula.

We note that the right side of (4.6) is zero if and only if $x_{i}=x_{i+n}$ for each $i$. Any finite collection of points on the circle may be treated by first adding all antipodal points, and then diagonalizing as in (4.6). The additional points may be suppressed by setting the corresponding $x_{i}$ equal to zero. We have proved the following lemma.

Lemma 4.3. Let $p_{1}, \cdots, p_{n}$ be points on a unit circle. Then the quadratic form $\Sigma_{i<j} d^{\prime}\left(p_{i}, p_{j}\right) x_{i} x_{j}$ is negative semidefinite on the byperplane $\Sigma_{i} x_{i}=0$.

Theorem 4.2. The great circle metric is semidefinite. The form $\Sigma_{i<j} d^{\prime}\left(p_{i}, p_{j}\right) x_{i} x_{j}$ will be negative definite on $\Sigma_{i} x_{i}=0$ unless there are two pairs of antipodal points among the $p_{i}$.

Proof (for the 2-sphere). Let $\Gamma$ be an arc of a great circle on the sphere, and let $p$ be a point which is neither an endpoint of $\Gamma$ nor antipodal to an endpoint. Let $\Gamma_{p}$ be the projection of $\Gamma$ via great circles onto the equator of $p$. We note that $|\Gamma|=1 / 4 \pi^{-1} \int\left|\Gamma_{p}\right| d \sigma(p)$. The integral is clearly invariant. To see that $1 / 4 \pi$ is the correct constant, choose $\Gamma$ to be a great circle.

Let $p_{1}, \cdots, p_{n}$ be distinct points on the sphere and let $\Gamma^{i j}$ be a minimal great arc joining $p_{i}$ and $p_{j}$. We note that

$$
\sum_{i<j} d^{\prime}\left(p_{i}, p_{j}\right) x_{i} x_{j}=\frac{1}{4 \pi} \int \sum_{i<j}\left|\Gamma_{p}^{i j}\right| x_{i} x_{j} d \sigma(p) .
$$


The right side of (4.7) is nonpositive by Lemma 4.3. It is clear from an examination of (4.6) that the form can be zero in a nontrivial way if and only if two pairs of antipodal points are among the $p_{i}$.

If in Theorem 4.1 we allow $b$ to be semidefinite, the right inequality of (4.2) can become equality. Two important examples are given by $d^{2}$ and $d^{\prime}$. See (2.1) for $d^{2}$; if $p_{1}, \cdots, p_{2 n}$ are such that $p_{i}$ and $p_{i+n}$ are antipodal, then $\Sigma_{i<j} d^{\prime}\left(p_{i}, p_{j}\right)$ $=1 / 4 \pi(2 n)^{2}$ and $c\left(d^{\prime}\right)=1 / 4 \pi$. The papers of Sperling [31] and Nielson [23] concern $d^{\prime}$. The latter paper uses the integral formula for arc length and is closely related to our work. Kelly obtains results of the same type inter alia in [17]; see also his papers [15] and [16] for results concerning the quadratic form $I(K, \mu)$ of $\$ 3$ and the Schoenberg embedding theorems.

We remark that if $b$ is semidefinite, then $b^{\gamma}$ is definite for $0<\gamma<1$.

5. A proof using spherical harmonics. In this section we give a proof (which uses the ideas of [24]) of the upper bound of Theorem 4.1 in the special case where $b(p, q)$ is the Euclidean distance from $p$ to $q$. In fact, we ultimately obtain the stronger inequalities (5.33) and (5.34).

The notation in this section is changed to conform to that of Chapters $\mathrm{X}$ and $\mathrm{XI}$ of [6]. We let $q_{1}, \cdots, q_{N}$ denote points on the unit sphere in $E^{p+2}, p \geq 0$, and set $t=1 / 2 p$. For $0<\lambda<2$ we define

$$
S(N, p, \lambda)=\max \sum_{i<j} d_{i j}^{\lambda}
$$

where $d_{i j}=\left|q_{i}-q_{j}\right|$. The upper bound of Theorem 4.1 now becomes

$$
s(N, p, \lambda) / N^{2}<2^{p-1+\lambda} \Gamma(1+1 / 2 p) \Gamma(1 / 2(p+1+\lambda)) / \pi^{1 / 2} \Gamma(p+1+1 / 2 \lambda) .
$$

For the moment we assume $p \geq 1$; the modifications required when $p=0$ will be given later.

The ultraspherical (or Gegenbauer) polynomials are defined by the generating function

$$
\left(1-2 x r+r^{2}\right)^{-t}=\sum_{m=0}^{\infty} C_{m}^{t}(x) r^{m}, \quad t \neq 0 .
$$

For example $C_{0}^{t}(x)=1, C_{1}^{t}(x)=2 t x$, etc.; they are orthogonal on $[-1,1]$ with weight $w(x)=\left(1-x^{2}\right)^{t-1 / 2}$. We shall write $(\alpha)_{m}=\Gamma(\alpha+m) / \Gamma(\alpha)$, and frequently make use of

$$
\Gamma(\alpha+m) / \Gamma(m) \sim m^{a}, \quad m \rightarrow \infty
$$

and

$$
\sqrt{\pi} \Gamma(2 z)=2^{2 z-1} \Gamma(z) \Gamma(z+1 / 2)
$$


Lemma 5.1. For $0<\lambda<2,0<r \leq 1$, and $-1 \leq x \leq 1$ let

$$
f(x)=\left(1+r^{2}-2 r x\right)^{\lambda / 2} \sim \sum_{m=0}^{\infty} a_{m}(r) C_{m}^{t}(x), a_{m}(r)=a_{m}(r, \lambda),
$$

be the formal expansion of $f(x)$ in terms of ultraspherical polynomials. Then (i) the series converges to $f(x)$ in $[-1,1]$, and (ii) for $r<1$ we bave $a_{0}(r)>0$ but $a_{m}(r)<0, m \geq 1$. Hence $a_{0}(1) \geq 0$ and $a_{m}(1) \leq 0, m \geq 1$.

Proof. If $r \neq 1$, then $f(x)$ is analytic on $[-1,1]$ and hence by Theorem 9.1.1, p. 243 of $[34]$ the series converges to $f(x)$ in the interior of the ellipse $|x-1|+$ $|x+1|=\left(1+r^{2}\right) / r$ (consider $x$ as a complex variable). For $r=1, f(x)$ is continuous and $\left(1-x^{2}\right)^{t-1 / 2}|f(x)|$ is integrable, so by [18, p. 779] and [19, p. 168] the series on the right of $(5.4)$ is Cesàro $(C, k)$ summable to $f(x)$ in the closed interval $[-1,1]$ for $k$ sufficiently large. Hence to show (i) it suffices to show that the series converges. We do this directly for any $r, 0<r \leq 1$.

By $[6$, p. 175 (iii) (11)] we have the Rodrigues' formula

$$
2^{m} m !(t+1 / 2)_{m}\left(1-x^{2}\right)^{t-1 / 2} C_{m}^{t}(x)=(-1)^{m}(2 t)_{m} D^{m}\left[\left(1-x^{2}\right)^{m+t-1 / 2}\right] .
$$

We obtain from orthogonality and repeated integration by parts,

$$
\begin{aligned}
b a_{m}(r) & =\int_{-1}^{1}\left(1+r^{2}-2 r x\right)^{\lambda / 2}\left(1-x^{2}\right)^{t-1 / 2} C_{m}^{t}(x) d x \\
& =\frac{(-1)^{2 m}(2 t)_{m}}{2^{m} m !(t+1 / 2)_{m}} \int_{-1}^{1}\left(1-x^{2}\right)^{m+t-1 / 2} D^{m}\left(1+r^{2}-2 r x\right)^{\lambda / 2} d x
\end{aligned}
$$

where $b$ is the orthonormalization constant (see $[6$, p. $236(26)]$ or $[34$, p. 82])

$$
b=\int_{-1}^{1}\left(1-x^{2}\right)^{t-1 / 2}\left(C_{m}^{t}(x)\right)^{2} d x=2^{1-2 t} \pi \Gamma(t)^{-2} \frac{\Gamma(m+2 t)}{(m+t) \Gamma(m+1)} .
$$

Since

$$
D^{m}\left(1+r^{2}-2 r x\right)^{\lambda-2}=(2 r)^{\lambda / 2} \frac{\Gamma(m-\lambda / 2)}{\Gamma(-\lambda / 2)}\left(\frac{1+r^{2}}{2 r}-x\right)^{\lambda / 2-m}
$$

(ii) is immediate. By [6, p. 207 (7)]

$$
\max _{-1 \leq x \leq 1}\left|C_{m}^{t}(x)\right|=C_{m}^{t}(1)=(2 t)_{m} / m !, \quad t>0 .
$$

So for $m \geq 1$ the facts that $(1-x)<\left(\left(1+r^{2}\right) / 2 r-x\right)$ and 


$$
\int_{-1}^{1}(1+x)^{p-1}(1-x)^{q-1} d x=2^{p+q-1} \Gamma(p) \Gamma(q) / \Gamma(p+q)
$$

yield

$$
b a_{m}(r) \leq \frac{(2 t)_{m}(2 r)^{\lambda / 2} \Gamma(m-\lambda / 2) 2^{2 t+\lambda / 2+m} \Gamma(t+\lambda / 2+1 / 2) \Gamma(m+t+1 / 2)}{2^{m} m !(t+1 / 2)_{m} \Gamma(-\lambda / 2) \Gamma(m+2 t+\lambda / 2+1)}
$$

with equality only when $r=1$. Now by (5.2) $b \gg m^{p-2}$, so by (5.2), (5.8), and (5.10),

$\left|a_{m}(r) C_{m}^{t}(x)\right| \ll \exp [(\log m)(2-p+p-1+p-1-t-1 / 2-\lambda / 2+t+1 / 2-p-\lambda / 2-1)]$

$$
=m^{-1-\lambda} \text {, }
$$

and $\Sigma_{m}-1-\lambda$ converges, so the proof is complete.

Lemma 5.2.

$$
-a_{m}(1)=\frac{2^{2 t+\lambda}}{\sqrt{\pi}} \frac{\lambda}{2} \Gamma(t)(m+t) \frac{\Gamma(m-\lambda / 2) \Gamma(t+\lambda / 2+1 / 2)}{\Gamma(1-\lambda / 2) \Gamma(m+2 t+\lambda / 2+1)}
$$

Proof. This follows from (5.10) and (5.3).

Definition. Let $\theta_{i}\left(0 \leq \theta_{i} \leq \pi, i=1, \ldots, p\right)$ and $\theta_{p+1}\left(0 \leq \theta_{p+1}<2 \pi\right)$ be called the generalized latitudes and longitude of a point $q_{\theta}$ on the unit sphere in $E^{p+2}$ where

$$
\begin{aligned}
q_{\theta}=\left(\cos \theta_{1}, \sin \theta_{1} \cos \theta_{2}, \sin \theta_{1} \sin \theta_{2} \cos \theta_{3},\right. \\
\cdots, \sin \theta_{1} \sin \theta_{2} \cdots \sin \theta_{p} \cos \theta_{p+1}, \\
\left.\quad \sin \theta_{1} \sin \theta_{2} \cdots \sin \theta_{p} \sin \theta_{p+1}\right) .
\end{aligned}
$$

Define $[\theta, \phi]=\left[\left(\theta_{1}, \ldots, \theta_{k}\right),\left(\phi_{1}, \ldots, \phi_{k}\right)\right]$ inductively by

$$
\begin{aligned}
{\left[\left(\theta_{1}\right),\left(\phi_{1}\right)\right]=} & \cos \left(\theta_{1}-\phi_{1}\right), \\
{\left[\left(\theta_{1}, \ldots, \theta_{k}\right),\left(\phi_{1}, \ldots, \phi_{k}\right)\right]=} & \cos \theta_{1} \cos \phi_{1}+\left(\sin \theta_{1} \sin \phi_{1}\right) \\
& \cdot\left[\left(\theta_{2}, \ldots, \theta_{k}\right),\left(\phi_{1}, \ldots, \phi_{k}\right)\right] .
\end{aligned}
$$

Let $\psi_{1}$ be the angle made by $q_{\theta}$ and $q_{\phi}$ at the center of the sphere. Then $\cos \psi_{1}=\left[\left(\theta_{1}, \ldots, \theta_{p+1}\right),\left(\phi_{1}, \cdots, \phi_{p+1}\right)\right]$. In general, set

$$
\cos \psi_{r}=\left[\left(\theta_{r}, \cdots, \theta_{p+1}\right),\left(\phi_{r}, \cdots, \phi_{p+1}\right)\right]
$$

the right side of $(5.13)$ is clearly in $[-1,1]$ since it equals

$$
\left[\left(\pi / 2, \cdots, \pi / 2, \theta_{r}, \cdots, \theta_{p+1}\right),\left(\pi / 2, \ldots, \pi / 2, \phi_{r}, \cdots, \phi_{p+1}\right)\right] .
$$

In what follows we often write $j(i)$ for $j_{i}$. Recall the addition formulae for 
Legendre polynomials $P_{n}(x)=C_{n}^{1 / 2}(x)[6$, p. 183] and for ultraspherical polynomials $C_{n}^{t}(x)\left[6\right.$, p. 178 (the $2^{m}$ should be $\left.\left.2^{2 m}\right)\right]$ :

$$
P_{n}(\cos \theta \cos \phi+\sin \theta \sin \phi \cos \psi)
$$

$$
=P_{n}(\cos \theta) P_{n}(\cos \phi)+2 \sum_{m=1}^{n} \frac{(n-m) !}{(n+m) !} P_{n}^{m}(\cos \theta) P_{n}^{m}(\cos \phi) \cos n \psi \text {. }
$$

$$
C_{n}^{t}(\cos \theta \cos \phi+\sin \theta \sin \phi \cos \psi)
$$

$$
=\sum_{m=0}^{n} 2^{2 m}(2 t+2 m-1)(n-m) ! \frac{\left[(t)_{m}\right]^{2}}{(2 t-1)_{n+m+1}}
$$

$$
\text { - }(\sin \theta)^{m} C_{n-m}^{t+m}(\cos \theta)(\sin \phi)^{m} C_{n-m}^{t+m}(\cos \phi) C_{m}^{t-1 / 2}(\cos \psi) \text {. }
$$

In (5.14) the $P_{n}^{m}$ are the associated Legendre polynomials of order $m$. Note that all coefficients in (5.14) and (5.15) are nonnegative. By (5.12) and repeated applications of (5.15),

$$
\begin{aligned}
& C_{j(0)}^{t}\left(\left[\left(\theta_{1}, \cdots, \theta_{p+1}\right),\left(\phi_{1}, \cdots, \phi_{p+1}\right)\right]\right) \\
& =\sum_{j(1)=0}^{j(0)} \cdots \sum_{j(p-1)=0}^{j(p-2)} b(j(0), j(1), t) \\
& \cdots b(j(n-3), j(n-2), t-1 / 2(p-2)) f(\theta) f(\phi) \cdot g
\end{aligned}
$$

where $b(j(i), j(i+1), t-1 / 2(i-1)) \geq 0$,

$$
\begin{array}{r}
f(\theta)=\left(\sin \theta_{1}\right)^{j(1)} \cdots\left(\sin \theta_{p-1}\right)^{j(p-1)} C_{j(0)-j(1)}^{t+j(1)}\left(\cos \theta_{1}\right) \\
\cdots C_{j(p-2)-j(p-1)}^{t-1 /(p-2)+j(p-1)}\left(\cos \theta_{p-1}\right),
\end{array}
$$

and (by (5.14))

$$
\begin{aligned}
g & =C_{j(p-1)}^{t-1 /(p-1)}\left(\cos \psi_{p}\right)=P_{j(p-1)}\left(\cos \theta_{p} \cos \phi_{p}+\sin \theta_{p} \sin \phi_{p} \cos \left(\theta_{p+1}-\phi_{p+1}\right)\right) \\
= & P_{j(p-1)}\left(\cos \theta_{p}\right) P_{j(p-1)}\left(\cos \phi_{p}\right) \\
& +2 \sum_{i=1}^{j(p-1)} \frac{(j(p-1)-i) !}{(j(p-1)+i) !} a_{i}\left(\theta_{p}, \theta_{p+1}\right) a_{i}\left(\phi_{p}, \phi_{p+1}\right) \\
& +2 \sum_{i=1}^{j(p-1)} \frac{(j(p-1)-i) !}{(j(p-1)+i) !} b_{i}\left(\theta_{p}, \theta_{p+1}\right) b_{i}\left(\phi_{p}, \phi_{p+1}\right)
\end{aligned}
$$


where $a_{i}\left(\theta_{p}, \theta_{p+1}\right)=P_{j(p-1)}^{i}\left(\cos \theta_{p}\right) \cos j(p-1) \theta_{p+1}$ and $b_{i}\left(\theta_{p}, \theta_{p+1}\right)=$ $P_{j(p-1)}^{i}\left(\cos \theta_{p}\right) \sin j(p-1) \theta_{p+1}$. Hence

$$
C_{j(0)}^{t}\left(\left[\left(\theta_{1}, \ldots, \theta_{p+1}\right),\left(\phi_{1}, \ldots, \phi_{p+1}\right]\right]\right)=\sum_{i=1}^{l} c_{i} b_{i}(\theta) b_{i}(\phi)
$$

for appropriate $b_{i}$, with $c_{i} \geq 0$.

Lemma 5.3. Let $q_{\nu}=\left(\theta_{1}^{(\nu)}, \ldots, \theta_{p+1}^{(\nu)}\right), \nu=1, \ldots, N$, be $N$ points on the unit $p+2$ sphere and let $\gamma_{\mu \nu}$ be the smallest angle between $q_{\mu}$ and $q_{\nu}$ Then

$$
\sum_{\mu<\nu} C_{m}^{t}\left(\cos \gamma_{\mu \nu}\right) \geq-1 / 2 N(2 t)_{m} / m !
$$

Proof. This follows from (5.8) and

$$
\sum_{\mu=1}^{N .} \sum_{\nu=1}^{N} C_{m}^{t}\left(\cos \gamma_{\mu \nu}\right)=\sum_{i=1}^{l} c_{i}\left[\sum_{\mu=1}^{N} b_{i}\left(\theta^{(\mu)}\right)\right]^{2} \geq 0
$$

Lemma 5.4. If the real part of $c-a-b$ is positive,

$$
{ }_{2} F_{1}[a, b ; c ; 1]=1+\frac{\Gamma(c)}{\Gamma(a) \Gamma(b)} \sum_{m=1}^{\infty} \frac{\Gamma(m+a) \Gamma(m+b)}{m ! \Gamma(m+c)}=\frac{\Gamma(c) \Gamma(c-a-b)}{\Gamma(c-a) \Gamma(c-b)} .
$$

Proof. See [35, pp. 281-282].

We can now begin the proof of the theorem. By Lemmas (5.1) and (5.3),

$$
\begin{aligned}
\sum & =\left(\begin{array}{l}
N \\
2
\end{array}\right)^{-1} \sum_{\mu<\nu}\left|q_{\mu}-q_{\nu}\right|^{\lambda}=\left(\begin{array}{l}
N \\
2
\end{array}\right)^{-1} \sum_{\mu<\nu}\left[2\left(1-\cos \gamma_{\mu \nu}\right)\right]^{\lambda / 2} \\
& =a_{0}(1)+\left(\begin{array}{l}
N \\
2
\end{array}\right)^{-1} \sum_{m=1}^{\infty}\left|a_{m}(1)\right| \cdot(-1) \sum_{\mu<\nu} c_{m}^{t}\left(\cos \gamma_{\mu \nu}\right)
\end{aligned}
$$

$$
\begin{aligned}
& \leq a_{0}(1)+\left(\begin{array}{l}
N \\
2
\end{array}\right)^{-1} \sum_{m=1}^{\infty}\left(-a_{m}(1)\right) 1 / 2 N(2 t)_{m} / m ! \\
& \leq a_{0}(1)-\frac{1}{N-1} \sum_{m=1}^{\infty} a_{m}(1)(2 t)_{m} / m !=U .
\end{aligned}
$$

By Lemma 5.2,

$$
U=\frac{2^{2 t+\lambda}}{\sqrt{\pi}} \frac{\lambda}{2} \frac{\Gamma(t) \Gamma(t+\lambda / 2+1 / 2)}{\Gamma(1-\lambda / 2)}\left[\frac{-t \Gamma(-\lambda / 2)}{\Gamma(2 t+\lambda / 2+1)}+\frac{V}{(N-1) \Gamma(2 t)}\right]
$$

where 


$$
\begin{aligned}
V= & \sum_{m=1}^{\infty} \frac{(m+t) \Gamma(m+2 t) \Gamma(m-\lambda / 2)}{\Gamma(m+1) \Gamma(m+2 t+\lambda / 2+1)} \\
= & \sum_{m=1}^{\infty} \frac{\Gamma(m-1+1+2 t) \Gamma(m-1+1-\lambda / 2)}{\Gamma(m) \Gamma(m-1+2 t+\lambda / 2+2)} \\
& +t\left[-\frac{\Gamma(2 t) \Gamma(-\lambda / 2)}{\Gamma(2 t+\lambda / 2+1)}+\frac{\Gamma(2 t) \Gamma(-\lambda / 2)}{\Gamma(2 t+\lambda / 2+1)}+\sum_{m=1}^{\infty} \frac{\Gamma(m+2 t) \Gamma(m-\lambda / 2)}{m ! \Gamma(m+2 t+\lambda / 2+1)}\right] \\
= & \frac{\Gamma(1+2 t) \Gamma(1-\lambda / 2) \Gamma(\lambda)}{\Gamma(\lambda / 2+1) \Gamma(2 t+\lambda+1)}-\frac{t \Gamma(2 t) \Gamma(-\lambda / 2)}{\Gamma(2 t+\lambda / 2+1)}+\frac{\Gamma(2 t) \Gamma(-\lambda / 2) t \Gamma(\lambda+1)}{\Gamma(\lambda / 2+1) \Gamma(2 t+\lambda+1)} \\
= & \frac{t \Gamma(2 t) \Gamma(-\lambda / 2)}{\Gamma(\lambda / 2+1) \Gamma(2 t+\lambda+1)}[2(-\lambda / 2) \Gamma(\lambda)+\Gamma(\lambda+1)]-\frac{t \Gamma(2 t) \Gamma(-\lambda / 2)}{\Gamma(2 t+\lambda / 2+1)}
\end{aligned}
$$

Hence by (5.3)

$$
\begin{aligned}
U & =\frac{2^{2 t+\lambda}}{\sqrt{\pi}} \frac{t \Gamma(t) \Gamma(t+\lambda / 2+1 / 2)}{\Gamma(1-\lambda / 2)} \frac{\Gamma(1-\lambda / 2)}{\Gamma(2 t+\lambda / 2+1)} \frac{N}{N-1} \\
& =\frac{2^{p+\lambda}}{\sqrt{\pi}} \frac{\Gamma(p / 2+1) \Gamma(p / 2+\lambda / 2+1 / 2)}{\Gamma(p+1+\lambda / 2)} \frac{N}{N-1}
\end{aligned}
$$

Now (5.1) will follow as soon as we show that the inequality in (5.19) is strict. Equality holds only if for every $m \geq 1$

$$
\sum_{\mu<\nu} C_{m}^{t}\left(\cos \gamma_{\mu \nu}\right)=-1 / 2 N(2 t)_{m} / m !
$$

Think of $q_{1}, \cdots, q_{N}$ as defining a linear operator $L$; i.e.,

$$
L(f(x))=\sum_{\mu<\nu} f\left(\cos \gamma_{\mu \nu}\right)
$$

Lemma 5.5. Let $k \geq 1$ be an integer. Then

$$
\sum_{m=1}^{k}(-1)^{m+1} \frac{\Gamma(2 k-m+t)}{m ! \Gamma(k-m+1) \Gamma(k-m+1+t)}=\frac{\Gamma(2 k+t)}{\Gamma(k+1) \Gamma(k+1+t)}
$$

for all complex $t$.

Proof. Both sides are polynomials in $t$ of degree $k-1$, so it suffices to show the inequality for $k$ distinct integral values of $t$. But for $t$ a positive integer, (5.21) is a special case of the well-known formula 


$$
\sum_{m=0}^{s}(-1)^{m}\left(\begin{array}{c}
s \\
m
\end{array}\right)\left(\begin{array}{c}
u-m \\
t
\end{array}\right)=\left(\begin{array}{c}
u-s \\
u-t
\end{array}\right)
$$

see for example [22, p. 252 (27)].

Lemma 5.6. Let the linear operator $L$ be defined by $L(1)=\left({ }_{2}^{N}\right)$ and $L\left(C_{n}^{t}(x)\right)$ $=-1 / 2 N(2 t)_{n} / n !$. Then for integers $k \geq 0$,

$$
L\left(x^{2 k}\right)=\left\{\Gamma(2 k) \Gamma(1+t) / 2^{2 k} \Gamma(k) \Gamma(k+1+t)\right\} N^{2}-1 / 2 N ;
$$

the right side of $(5.22)$ is to be replaced by its limit $(=1 / 2)$ when $k=0$.

Proof. The lemma is clearly true for $k=0$; assume it is true for all integers $k^{\prime}$ with $0 \leq k^{\prime}<k$. By $[6$, p. $175(18)]$

$$
C_{2 k}^{t}(x)=\sum_{m=0}^{k}(-1)^{m} \frac{\Gamma(2 k-m+t)}{\Gamma(t) \Gamma(m+1) \Gamma(2 k-2 m+1)}(2 x)^{2 k-2 m} .
$$

Thus

$$
\begin{aligned}
\frac{\Gamma(2 k+t)}{\Gamma(t)(2 k) !} 2^{2 k} L\left(x^{2 k}\right)= & \sum_{m=1}^{k}(-1)^{m+1} \frac{\Gamma(2 k-m+t)}{\Gamma(t) \Gamma(m+1) \Gamma(2 k-2 m+1)} 2^{2 k-2 m} L\left(x^{2(k-m)}\right) \\
& -1 / 2 N \frac{\Gamma(2 t+2 k)}{\Gamma(2 t)(2 k) !} .
\end{aligned}
$$

By the induction hypothesis, (5.8), and Lemma 5.5 the result is established.

It follows from Lemma 5.6 that $\lim _{k \rightarrow \infty} L\left(x^{2 k}\right)=-1 / 2 N<0$ if $(5.20)$ is valid for $m \geq 1$. But

$$
L\left(x^{2 k}\right)=\sum_{\mu<\nu} \cos ^{2 k} \gamma_{\mu \nu} \geq 0 .
$$

Thus the theorem is proved when $p \geq 1$. For $p=0$ the details are quite similar. Let $q_{\mu}=e^{i \phi_{\mu}}$. Then

$$
\begin{aligned}
& \left(\begin{array}{l}
N \\
2
\end{array}\right)^{-1} \sum_{\mu<\nu}\left|e^{i \phi_{\mu}}-e^{i \phi_{\nu} \lambda}\right|^{\lambda}=\left(\begin{array}{l}
N \\
2
\end{array}\right)^{-1} \sum_{\mu<\nu}\left|2 \sin \frac{\phi_{\mu}-\phi_{\nu}}{2}\right|^{\lambda} \\
& =a_{0}+2 \sum_{m=1}^{\infty}\left(\begin{array}{l}
N \\
2
\end{array}\right)^{-1} a_{m} \sum_{\mu<\nu} \cos m\left(\phi_{\mu}-\phi_{\nu}\right) \\
& \leq a_{0}-\frac{2}{N-1} \sum_{m=1}^{\infty} a_{m}=U
\end{aligned}
$$


since $[24$, p. 32, Lemma IV]

$$
\sum_{\mu<\nu} \cos m\left(\phi_{\mu}-\phi_{\nu}\right) \geq-1 / 2 N \text {. }
$$

Also by [24], $a_{0}=\Gamma(1+\lambda) / \Gamma^{2}(1+\lambda / 2)$ and

$$
\begin{aligned}
a_{m} & =-\sin \frac{\pi \lambda}{2} \Gamma(1+\lambda) \Gamma(m-\lambda / 2) / \pi \Gamma(1+\lambda / 2+m) \\
& =(-1)^{m} \Gamma(1+\lambda) / \Gamma(1+\lambda / 2-m) \Gamma(1+\lambda / 2+m) .
\end{aligned}
$$

The sum $U$ is again evaluated by Lemma 5.4 and by the aid of (5.3) we find that (5.1) is also valid for $p=0$, provided strict inequality holds for some $m \geq 1$ in (5.25). But unless this is the case, we can use the identity

$$
\sum_{m=1}^{M} \cos m x=-1 / 2+1 / 2[\sin (2 M+1) 1 / 2 x] /[\sin 1 / 2 x]=-1 / 2+B(M, x)
$$

to show

$$
-\frac{N}{2} M=\sum_{\mu<\nu}\left[-1 / 2+B\left(M,\left(\phi_{\mu}-\phi_{\nu}\right)\right)\right]
$$

which is false for $M$ sufficiently large. This completes the proof of the theorem.

The proof of this section appears more complicated than the earlier proof, but a more careful estimation of the hyperspherical sum $\Sigma$ of (5.19) will now yield a stronger result than (5.1).

First assume $p \geq 1$. Let $L$ be a linear operator on polynomials with $L(1)=$ $\left(\begin{array}{l}N \\ 2\end{array}\right)$ and write

$$
L\left(C_{n}^{t}(x)\right)=\left\{-1 / 2 N(2 t)_{n} / n !\right\}+\epsilon_{n} .
$$

We shall show that if $L$ is the operator defined by $q_{1}, \cdots, q_{N}$ then

$$
\left|\epsilon_{n}\right| \leq c_{0}(p) N^{(2 p-1) /(p+1)}, \quad 1 \leq n \leq c_{1}(p) N^{2 /(p+1)},
$$

is false. Here the $c_{i}(p)$ will denote certain positive constants depending only on $p$ which can be written down explicitly; similarly for $c_{i}(p, \lambda)$ and $c_{i}(\lambda)$.

Assume (5.27) is true and write

$$
L\left(x^{2 k}\right)=R(N, k, t)+\theta_{2, k}
$$

where $R(N, k, t)$ is the expression on the right of (5.22). Recall that $t=1 / 2 p$.

Then

$$
\theta_{2 k}=\sum_{m=1}^{k}(-1)^{m+1} 2^{-2 m} c_{k m} \theta_{2(k-m)}+2^{-2 k} \Gamma(t) \frac{\Gamma(2 k+1)}{\Gamma(2 k+t)} \epsilon_{2 k}
$$

where 


$$
c_{k m}=\frac{\Gamma(2 k+1)}{\Gamma(2 k+t)} \cdot \frac{\Gamma(2 k-m+t)}{\Gamma(2 k+1-2 m) \Gamma(m+1)} .
$$

Lemma 5.7. If $\delta_{0}=0$ and

$$
a_{n}=\sum_{\nu=1}^{n}(-1)^{\nu+1} 2^{-2 \nu} c_{n \nu} a_{n-\nu}+2^{-2 n} \delta_{n}
$$

then

$$
a_{n}=2^{-2 n} \sum_{\nu=1}^{n} b_{n \nu} \delta_{\nu}
$$

where

$$
b_{n \nu}=\frac{\Gamma(2 n+1)}{\Gamma(n-\nu+1) \Gamma(2 \nu+1)} \cdot \frac{\Gamma(2 \nu+t+1)}{\Gamma(n+\nu+t+1)} .
$$

Proof. This is clear for $n=0$. Assume true for all integers less than $n$. Then

$$
\begin{aligned}
a_{n} & =\sum_{\nu=1}^{n}(-1)^{\nu+1} 2^{-2 \nu} c_{n \nu} 2^{-2(n-\nu)} \sum_{j=1}^{n-\nu} b_{n-\nu, j} \delta_{j}+2^{-2 n} \delta_{n} \\
& =-\sum_{j=1}^{n-1} 2^{-2 n} \delta_{j} \sum_{\nu=1}^{n-j}(-1)^{\nu} c_{n \nu} b_{n-\nu, j}+2^{-2 n} \delta_{n} \\
& =\sum_{j=1}^{n} 2^{-2 n} b_{n j} \delta_{j}-\sum_{j=1}^{n-1} 2^{-2 n} \delta_{j} \sum_{\nu=0}^{n-j}(-1)^{\nu} c_{n \nu} b_{n-\nu, j}
\end{aligned}
$$

The vanishing of the sum on $\nu$ is proved in the same way as in Lemma 5.5, and by the same identity.

It follows immediately from Lemma 5.7 that

$$
\left|\theta_{2 k}\right| \leq c_{2}(p) \max _{\nu=1}^{k} \frac{\Gamma(2 \nu+1)}{\Gamma(2 \nu+t)} \epsilon_{2 \nu^{\circ}}
$$

From (5.22) and estimates of the Stirling type,

$$
R(N, k, t)<-1 / 4 N
$$

whenever

$$
k \geq c_{3}(p) N^{2 /(p+1)}=K_{0} .
$$

Now if $k=\left[K_{0}\right]+1$ and $c_{1}(p) \geq 4 c_{3}(p)$, 


$$
\begin{aligned}
L\left(x^{2 k}\right) & <-1 / 4 N+c_{4}(p) c_{0}(p) N^{(2 p-1) /(p+1)}\left(N^{2 /(p+1)}\right)^{1-t} \\
& <-1 / 4 N+c_{4}(p) c_{0}(p) N
\end{aligned}
$$

and we have $L\left(x^{2 k}\right)<0$ for $c_{0}(p) c_{4}(p)<1 / 4$. But this is a contradiction. Hence (5.27) is false for an appropriate $c_{0}(p)$ and the estimate in (5.19) holds with $U$ decreased by

$$
\left(\begin{array}{l}
N \\
2
\end{array}\right)^{-1} \Gamma(t)^{-1}\left|a_{m}(1)\right| c_{0}(p) N^{(2 p-1) /(p+1)}
$$

for some $m$ satisfying $1 \leq m \leq c_{5}(p) N^{2 /(p+1)}$. Since by (5.10)

$$
\left|a_{m}(1)\right| \geq c_{6}(p, \lambda) \lambda(2-\lambda)_{m}^{-\lambda-p}
$$

we have

$$
s(N, p, \lambda) \leq c(p+1 ; \lambda) N^{2}-c_{7}(p, \lambda) \lambda(2-\lambda) N^{-(2 \lambda+1) /(p+1)} .
$$

When $p=0$ we use a different technique.

Lemma 5.8. Given real numbers $\theta_{1}, \cdots, \theta_{l}$ and an integer $T>0$, there is an integer $m$ with $1 \leq m \leq 2 T^{l}$ such that

$$
\left\|m \theta_{i}\right\|<T^{-1}, \quad i=1, \ldots, l,
$$

where $\|x\|$ denotes the distance from $x$ to the nearest integer.

Proof. This is an easy consequence of the pigeonhole principle. A proof is given, e.g., in Volume 2 of [20, p. 133].

Lemma 5.9. Under the above bypothesis there is an integer $m$ satisfying $1 \leq m \leq 2 \cdot 8^{l}$ such that

$$
\sum_{i=1}^{l} \cos m \theta_{i} \geq 2^{-1 / 2} l
$$

Proof. Choose $m$ so that $\left\|m \theta_{i} / 2 \pi\right\| \leq 1 / 8$ for $i=1, \ldots, l$.

Now let $l=\left(\begin{array}{c}N \\ 2\end{array}\right)$ and let $\theta_{1}, \cdots, \theta_{l}$ be the $l$ numbers $\phi_{\mu}-\phi_{\nu}$ where $\mu<\nu$. Choose $m$ as in Lemma 5.8. Then $U$ can be decreased by

$$
2\left(\begin{array}{l}
N \\
2
\end{array}\right)^{-1}\left|a_{m}\right| \cdot 2^{-1 / 2}\left(\begin{array}{l}
N \\
2
\end{array}\right)
$$

where $m \leq 2 \cdot 8^{l}$. It follows that

$$
s(N, 0, \lambda) \leq c(1, \lambda) N^{2}-c_{8}(\lambda) \lambda(2-\lambda) \exp \left(-c_{9}(\lambda) N^{2}\right) .
$$


6. Calculation of transfinite diameters and curvature. Let the points $p_{\mu}$ lie in a compact set $K$. If for $\lambda \geq 0$ we define $d_{N}^{(\lambda)}(K)=d_{N}^{(\lambda)}$ by

$$
\left(d_{N}^{(\lambda)}\right)^{\lambda}=\left(\begin{array}{l}
N \\
2
\end{array}\right)^{-1} \max \sum_{\mu<\nu}\left|p_{\mu}-p_{\nu}\right|^{\lambda}
$$

then it is well known [24] that $d_{2}^{(\lambda)} \geq d_{3}^{(\lambda)} \geq \cdots \geq 0$. Hence $d_{\infty}^{(\lambda)}=\lim _{N \rightarrow \infty} d_{N}^{(\lambda)}$ exists, and by letting $\lambda \rightarrow 0$ we get $d_{2}^{(0)} \geq d_{3}^{(0)} \geq \cdots \geq 0$. Hence $d_{\infty}=\lim _{N \rightarrow \infty} d_{N}^{(0)}$ also exists. We write $d_{N}=d_{N}^{(0)}$ and call $d_{\infty}^{(\lambda)}, d_{\infty}$ the $\lambda_{\text {th }}$ transfinite diameter of $K$ and the (usual) transfinite diameter of $K$ respectively.

Theorem 6.1. If $S_{n} \subseteq E^{n}$ is the surface of the n-dimensional unit spbere, $n=p+2 \geq 2$, then

$$
\begin{array}{ll}
d_{\infty}\left(S_{n}\right) & \\
\quad=\exp \left[1+\frac{1}{3}+\frac{1}{5}+\cdots+\frac{1}{n-3}-\frac{1}{2}\left(1+\frac{1}{2}+\frac{1}{3}+\cdots+\frac{1}{n-2}\right)\right], & n \text { even, } \\
\quad=2 \exp \left[\frac{1}{2}+\frac{1}{4}+\cdots+\frac{1}{n-3}-\frac{1}{2}\left(1+\frac{1}{2}+\frac{1}{3}+\cdots+\frac{1}{n-2}\right)\right], & n \text { odd, }
\end{array}
$$

with the convention that an empty sum is 0 .

Proof. It is not hard to use the "uniform distribution technique" of [24] to show that the expressions of (6.2) are lower bounds for $d_{\infty}\left(S_{n}\right)$. The real problem is to show that they are upper bounds. Let $\sigma(\lambda)$ be the expression on the right of (5.1). Then

$$
d_{\infty}\left(S_{n}\right)=\lim _{N \rightarrow \infty} d_{N}\left(S_{n}\right) \leq \lim _{\lambda \rightarrow 0} \sigma(\lambda)^{1 / \lambda}
$$

where

$$
d_{N}\left(S_{n}\right)=\lim _{\lambda \rightarrow 0}\left[s(N, p, \lambda) / N^{2}\right]^{1 / \lambda} .
$$

Now expansion of the $\Gamma$ functions yields $\sigma(\lambda)=$

$$
\Gamma\left(\frac{n}{2}\right) 2^{1 / 2 n-1} \cdot \frac{(n-3+\lambda)(n-5+\lambda) \cdots(1+\lambda) \Gamma(1+\lambda)}{(n-2+\lambda / 2)(n-3+\lambda / 2) \cdots(1+\lambda / 2) \Gamma^{2}(1+\lambda / 2)}
$$

or

$$
\frac{2^{1 / 2(n-1)}}{\sqrt{\pi}} \Gamma(n / 2) \cdot \frac{(n-3+\lambda)(n-5+\lambda) \cdots(2+\lambda)}{(n-2+\lambda / 2)(n-3+\lambda / 2) \cdots(3+\lambda / 2)} \cdot \frac{2^{\lambda}}{(1+\lambda / 2)}
$$

depending upon whether $n$ is even or odd. We can use the factors independent of $\lambda$ to put every factor involving $\lambda$ in the form $(1+a \lambda)$. Then (6.3) can be easily computed since 


$$
\lim _{\lambda \rightarrow 0}(1+\alpha \lambda)^{1 / \lambda}=e^{a} \text { and } \lim _{\lambda \rightarrow 0}\left[\Gamma(1+\lambda) / \Gamma^{2}(1+\lambda / 2)\right]^{1 / \lambda}=1
$$

The result follows.

Theorem 6.2. $d_{\infty}\left(S_{n}\right)=\sqrt{2}+Q_{1 / n}$ and (as this suggests) $d_{\infty}(U)=\sqrt{2}$ where $U$ is the surface of the unit sphere in Hilbert space.

Proof. The first statement follows readily from Theorem 6.1. For the second, $\sqrt{2} \leq d_{N}(U)$ is a consequence of the existence of $N$ unit orthonormal vectors in $U$ for any $N$. On the other hand, if we use (6.2) and (6.3) to calculate $\lim _{p \rightarrow \infty}$ in (5.1) (note that the left side is nondecreasing in $p$ ) we find that for $\lambda>0$

$$
s(N, \infty, \lambda) / N^{2} \leq 2^{\lambda / 2-1} \text {, }
$$

so

$$
d_{\infty} \leq d_{\infty}^{(\lambda)} \leq d_{N}^{(\lambda)}(U) \leq \sqrt{2}[N /(N-1)]^{1 / \lambda} .
$$

By taking $\lambda=N^{-1 / 2}$ and letting $N \rightarrow \infty$, the result follows.

$A$ related fact (proof omitted) is that the side of the regular simplex inscribed in $S_{n}$ tends to $\sqrt{2}$ as $n \rightarrow \infty$. That $d_{\infty}(U)=\sqrt{2}$ has already been proved by Hille [12].

Next, we propose a new purely metric definition of curvature (see [4, pp. 7489], [21, pp. 124-125] for a discussion of various such definitions). Let $M$ be a metric space with metric $d(x, y)$ and $S_{\rho}$ the sphere of radius $\rho$ about some $P \epsilon$ M. Let $0 \leq \lambda<2$ and set

$$
d_{N}^{(\lambda)}(\rho ; P)=d_{N}^{(\lambda)}=\left[\left(\begin{array}{l}
N \\
2
\end{array}\right)^{-1} \sup \sum_{1 \leq i<i \leq N} d^{\lambda}\left(p_{i}, p_{j}\right)\right]^{1 / \lambda}
$$

where the supremum is over all $p_{i} \in S_{\rho}, 1 \leq i \leq N$. Then $d_{N}^{(\lambda)}$ is nonincreasing, so $\lim _{N \rightarrow \infty} d_{N}^{(\lambda)}=d^{(\lambda)}$ exists.

Definition. If for some $n \geq 2$

$$
\pm \kappa^{2}=\lim _{\rho \rightarrow 0} \frac{6(2 n-2+\lambda)}{(n-1) \rho^{2}}\left(1-\frac{d^{(\lambda)}(\rho ; P)}{\rho \gamma(\lambda, n)}\right)
$$

where

$$
\gamma(\lambda, n)=\left[\frac{\Gamma(\lambda+n-1) \Gamma(n / 2)}{\Gamma(1 / 2(\lambda+n)) \Gamma(n-1+\lambda / 2)}\right]^{1 / \lambda},
$$

we say that as a space of dimension $n, M$ has a $\lambda$-curvature of $\kappa$ at $P$, the curvature being positive or negative according to the \pm sign. 
We conjecture that $\kappa=\kappa(P)$ lies between the minimal and maximal values of $\sqrt{K}$ where $K=K(P ;(\xi, \eta))$ is the Riemannian curvature of $M$ at $P$ in the two dimensional direction $(\xi, \eta)$ [21, pp. 125-127; pp. 136-137]; here it is assumed that $M$ is a sufficiently smooth manifold of dimension $n \geq 2$. This can be shown for $n \geq 2, M$ Euclidean (i.e., $k \equiv 0$ ) by the methods of this section and for $M$ of constant curvature, $n=2$, and $0 \leq \lambda \leq 1$ by the methods of $\$ 2$.

Acknowledgement. The authors are grateful to Richard Askey of the University of Wisconsin for pointing out to them the hyperspherical generalization of the addition theorem for Legendre functions. They also thank L. Fejes Tóth for the references [8], [23], and [31]. Finally, they thank the referee for pointing out many misprints and obscurities in an earlier version of this paper.

\section{REFERENCES}

1. R. Alexander, On the sum of distances between $n$ points on a sphere, Acta Math. Acad. Sci. Hungar. 23 (1972), 443-448.

2. G. Björck, Distributions of positive mass which maximize a certain generalized energy integral, Ark. Mat. 3 (1956), 255-269. MR 17, 1198.

3. L. M. Blumenthal, Distance geometries, University of Missouri Studies, Columbia, 1938.

4. - Theory and applications of distance geometry, Clarendon Press, Oxford, 1953. MR 14, 1009.

5. ——, A modern view of geometry, A Series of Undergraduate Books in Math., Freeman, San Francisco, Calif., 1961. MR 23 \#A3481.

6. A. Erdélyi, ed., Higher transcendental functions. Vol. 2, McGraw-Hill, New York, 1955. MR 15, 419.

7. L. Fejes Tóth, On the sum of distances determined by a pointset, Acta Math. Acad. Sci. Hungar. 7 (1956), 397-401. MR 21 \#5937.

8. - Über ein Punktverteilung auf der Kugel, Acta Math. Acad. Sci. Hungar. 10 (1959), 13-19. MR 21 \#4393.

9. B. Grünbaum and L. M. Kelly, Metrically homogeneous sets, Israel J. Math. 6 (1968), 183-197. MR $39 \# 6180$.

10. - Metrically homogeneous sets: corrigendum, Israel J. Math. 8 (1970), 93-95. MR $42 \# 3679$.

11. E. Hille, Analytic function theory. Vol. II, Introduction to Higher Math., Ginn, Boston, Mass., 1962. MR $34 \# 1490$.

12. - Some geometric extremal problems, J. Australian Math. Soc. 6 (1966), 122-128. MR $33 \# 6507$.

13. - Methods in classical and functional analysis, Addison-Wesley, Reading, Mass., 1971.

14. R. A. Johnson, Advanced Euclidean geometry: An elementary treatise on the geometry of the triangle and the circle (formerly titled: Modern geometry), Dover, New York, 1960. MR $22 \# 11289$.

15. J. B. Kelly, Metric inequalities and symmetric differences, Inequalities, II (Proc. Second Sympos., U. S. Air Force Acad., Colo. 1967), Academic Press, New York, 1970, pp. 193-212. MR $41 \# 9192$. 
16. J. B. Kelly, Hypermetric spaces and metric transforms, Inequalities, III, O. Shisha (editor), Academic Press, New York, 1972, pp. 149-158.

17. - Combinatorial in equalities, Combinatorial Structures and Their Applications (R. Guy, H. Hanani, N. Sauer, and J. Schonheim, eds.), Gordon and Breach, New York, 1970, pp. 201-207.

18. E. Kogbetliantz, Sur la sommation de séries ultrasphériques, C. R. Acad. Sci. (1917), 626-628, 778-780.

19. - Recherches sur la sommabilité des séries ultrasphériques par la méthode des moyennes arithmétiques, J. Math. 3 (1924), 107-187.

20. E. Landau, Vorlesungen über Zahlentheorie. Vol. 2, Hirzel, Leipzig, 1927.

21. D. Laugwitz, Differentialgeometrie, Teubner, Stuttgart, 1960; English transl., Differential and Riemannian geometry, Academic Press, New York, 1965. MR 22 \#7061; 30 \#2406.

22. E. Netto, Lehrbuch der Combinatorik, Teubner, Leipzig, 1901.

23. F. Nielson, Om summen af afstandene mellem $n$ punkter $\mathrm{pa}^{\circ}$ en Kugleflade, Nordisk Mat. Tidskr. 13 (1965), 45-50.

24. G. Pólya and G. Szegö, Über den transfiniten Durchmesser (Kapazitätskonstante) von ebenen und räumlichen Punktmengen, J. Reine Angew. Math 165 (1931), 4-49.

25. I. J. Schoenberg, Remarks to Maurice Fréchet's article "Sur la définition axiomatique d'une classe d'espaces vectoriels distanciés applicables vectoriellment sur l'espace de Hilbert," Ann. of Math. 36 (1935), 724-732.

26. - On certain metric spaces arising from Euclidean spaces by a change of metric and their imbedding in Hilbert space, Ann. of Math. 38 (1937), 787-793.

27. Metric spaces and positive definite functions, Trans. Amer. Math. Soc. 44 (1938), 522-536.

28. Metric spaces and completely monotone functions, Ann. of Math. 39 (1938), 811-841.

29. - Positive definite functions on spheres, Duke Math. J. 9 (1942), 96-108. MR 3, 232.

30. I. Schur, Über die Verteilung der Wurzeln bei gewissen algebraischen Gleichungen mit ganzzahligen Koeffizienten, Math. Z. 1 (1918), 377-402.

31. G. Sperling, Lösung einer elementargeometrischen Frage von Fejes Tóth, Arch. Math. 11 (1960), 69-71. MR 22 \#2935.

32. K. B. Stolarsky, Sums of distances between points on a sphere, Proc. Amer. Math. Soc. 35 (1972), 547-549.

33. - Sums of distances between points on a sphere. II, Proc. Amer. Math. Soc. 41 (1973), 575-582.

34. G. Szegö, Orthogonal polynomials, 2nd rev. ed., Amer. Math. Soc. Colloq. Publ., vol. 23, Amer. Math. Soc., Providence, R. I., 1959. MR 21 \#5029.

35. E. T. Whittaker and G. N. Watson, A course of modern analysis, 4th ed., Cambridge Univ. Press, New York, 1962. MR 31 \#2375.

36. H. S. Witsenhausen, On the maximum of the sum of squared distances under a diameter constraint, Amer. Math. Monthly (to appear).

37. - Metric inequalities and the zonoid problem, Proc. Amer. Math. Soc. 40 (1973), 517-520.

DEPARTMENT OF MATHEMATICS, UNIVERSITY OF ILLINOIS, URBANA, ILLINOIS 61801 